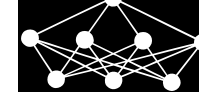

\title{
PRINCIPLES OF MOTOR RECOVERY IN POST-STROKE PATIENTS USING HAND EXOSKELETON CONTROLLED BY THE BRAIN-COMPUTER INTERFACE BASED ON MOTOR IMAGERY
}

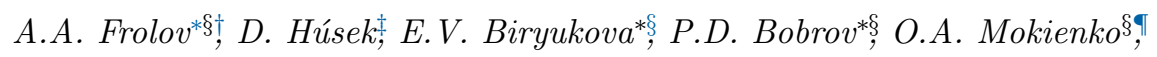

A.V. Alexandrov*

\begin{abstract}
Motor recovery in post-stroke and post-traumatic patients using exoskeleton controlled by the brain-computer interface (BCI) is a new and promising rehabilitation procedure. Its development is a multidisciplinary research which requires, the teamwork of experts in neurology, neurophysiology, physics, mathematics, biomechanics and robotics. Some aspects of all these fields of study concerning the development of this rehabilitation procedure are described in the paper. The description includes the principles and physiological prerequisites of BCI based on motor imagery, biologically adequate principles of exoskeleton design and control and the results of clinical application.
\end{abstract}

Key words: brain computer interface, motor imagery, post-stroke and post-traumatic patients, arm and hand exoskeleton, proportional derivative controller, motor synergy, clinical application

Received: October 31, 2016

DOI: $10.14311 / N N W .2017 .27 .006$

Revised and accepted: December 13, 2016

\section{Introduction}

According to the World Health Organization stroke incidences varies from 50 to 500 per 100000 population depending on the world region, and the rate of stroke mortalities amounts about $50 \%$ of stroke incidence [88]. Thus tens of millions people in the world suffer from the effects of stroke. After the treatment in rehabilitation clinics, almost a half of post-stroke patients can move only on a wheelchair, $15 \%$ can move in the room alone, $10 \%$ can go out and only $5 \%$ are able to go upstairs,

\footnotetext{
*A.A. Frolov - Corresponding author; E.V. Biryukova; P.D. Bobrov; A.V. Alexandrov; Institute of Higher Nervous Activity and Neurophysiology of the Russian Academy of Science, Russia, E-mail: aafrolov@mail.ru

${ }^{\dagger}$ A.A. Frolov; FEI, Technical University of Ostrava, Czech Republic

${ }^{\ddagger}$ D. Húsek; Institute of Computer Sience, the Czech Academy of Sciences, Czech Republic

$\S$ A.A. Frolov; E.V. Biryukova; P.D. Bobrov; O.A. Mokienko; Russian National Research Medical University, N.I. Pirogov, Russia

『O.A. Mokienko; Research Center of Neurology, Moscow, Russia
} 
although lower limb dysfunctions are less serious than those of the upper limbs [72]. The recovery of motor functions can be achieved by a variety of exercises, in particular by the repetition of active intended movements. However, for the majority of patients this is not enough [62]. This leads to the search for new technologies in stroke rehabilitation, including development of technical devices in order to increase the intensity of training, i.e. increasing the number of repetitions, and using bio-feedback. These technologies include training in virtual reality in conditions when movements are facilitated with robotic devices [62].

However, these methods require preserving at least a partial ability to make active movements of the paralyzed limb. In the case of a serious paresis or even paralysis, the promising method to stimulate brain plasticity is a motor imagery. As shown in many works $[35,35,51,51,52,84]$, motor imagery follows the same principles as the motor execution and, therefore, may stimulate the brain plasticity by the same mechanisms as the actual execution of movements. Monitoring of the movement imagery can be done with the help of brain-computer interface (BCI), which converts the EEG signals of the brain resulting from the movement imagery into the controlling commands for an external device. The BCI operator (patient) checks his own concentration on the movement imagery with the help of biofeedback.

Two approaches to usage of BCI in the rehabilitation procedures are discussed in the literature [23]. One approach is based only on a visual feedback when the signal of the recognized type of the imaginary movement is presented to the patient in the form of a label on the computer screen. The second approach requires addition of the kinesthetic feedback, when an imagery is accompanied by a passive movement executed with the help of exoskeleton. It may be supposed that the convergence of central motor command and peripheral signals on its execution is the best condition to activate the mechanisms of brain plasticity to restore motor functions.

Application of BCI and robots (orthoses, exoskeletons) in neurorehabilitation is a new and rapidly developing field of research. One of the impulses of this development was the discovery of plasticity in the functional topography of the primary motor cortex as a result of training [69]. It was shown relatively soon that the motor ability can be restored even after a few years after a stroke has occurred $[8,87]$. This resulted in the new principles for neurorehabilitation basing on intensive, regular and motivated movement execution [61]. Exoskeletons have become perfect technical devices to implement these principles. Different types of exoskeletons have been already actively used in the clinic, and the amount of their development in the last 10 years has grown exponentially [64]. Exoskeletons are often combined with a computer game in which the cursor movement relates to the movement of the operating point of the hand (via the joystick in the patient's hand). The patient with the help of visual feedback can solve the task of the given computer game. In some joints of exoskeletons passive spring mechanisms can be used for controlled removal of gravitation forces acting on the rehabilitated arms. This allows the patient to move his arm with minimal effort in the joints. In other exoskeletons active joints are used: the correct movement formation is achieved by their controllers. Some rehabilitation protocols (e.g. in computer games) contain adjustable parameters which allow for the formation of the correct movement. In other protocols, the physiotherapist initially performs a movement 
with patient's passive arm and after recording this movement it is reproduced with the help of exoskeleton or the patient performs the movement by the healthy arm and exoskeleton repeats this movement by paretic arm. Some examples of the most frequently used exoskeletons and the results of their clinical application are described in $[33,50]$.

In the reviews of the exoskeleton performance in neurorehabilitation two aspects are notable: The first one that is definitely positive is the possibility of the efficient use of exoskeletons in the post-stroke rehabilitation practice and the second one, rather negative, is the mostly emphasized fact, that progress in the development of more advanced exoskeletons suffers from the lack of understanding the principles of motion control by the central nervous system (CNS) [27]. What is worse is that even well-known principles of the motion control in living systems are only rarely used in the development of exoskeleton control systems.

In the following two principles of motion control by the BCI in human like style are discussed. The first is the control of the exoskeleton movement by the $\mathrm{P}$ (Proportional) D (Derivative) controller. The second is the formation of motor synergies, i.e. coordination of joint angles and torques in a multi-joint limb.

\section{Brain-Computer Interface}

\subsection{General principles}

General scheme of BCI is depicted in Fig. 1. It includes a system for the brain activity signals recording, then the interface that allows for their input into the computer, then the real-time classifier of data, next the real-time interface with an external device, and finally a feedback system (visual or/and proprioceptive) supplying information on the results of the command execution.

Two types of brain activity are used to create BCI: electrophysiological and hemodynamic.

Electrophysiological activity is due to electrochemical processes related to the transfer of information between neurons in the brain. There exist different variants how this activity can be acquired. For example, it can be registered in the form of electroencephalography (EEG) when electrodes are arranged at the head surface; in the form of magnetoencephalography (MEG) when magnetometers record magnetic field outside the head but produced by currents inside the brain; in the form of electrocorticography $(\mathrm{ECoG})$ when electrodes are located at the brain surface, but not in the brain tissue; and in the form of individual neuron activity when electrodes are located in the brain tissue.

Hemodynamic activity is due to an increase in oxygen delivery rate for the active areas of the brain as compared to inactive ones. This leads to local changes in the concentration ratio of oxyhemoglobin and deoxyhemoglobin. Changes in this ratio can be recorded by using functional magnetic resonance imaging (fMRI) or near-infrared spectroscopy (NIRS). There are already positive experiences of the implementation of the hybrid BCI, in which the assessment of a person's intention is based on the simultaneous analysis of electrical and hemodynamic brain activity [19]. 


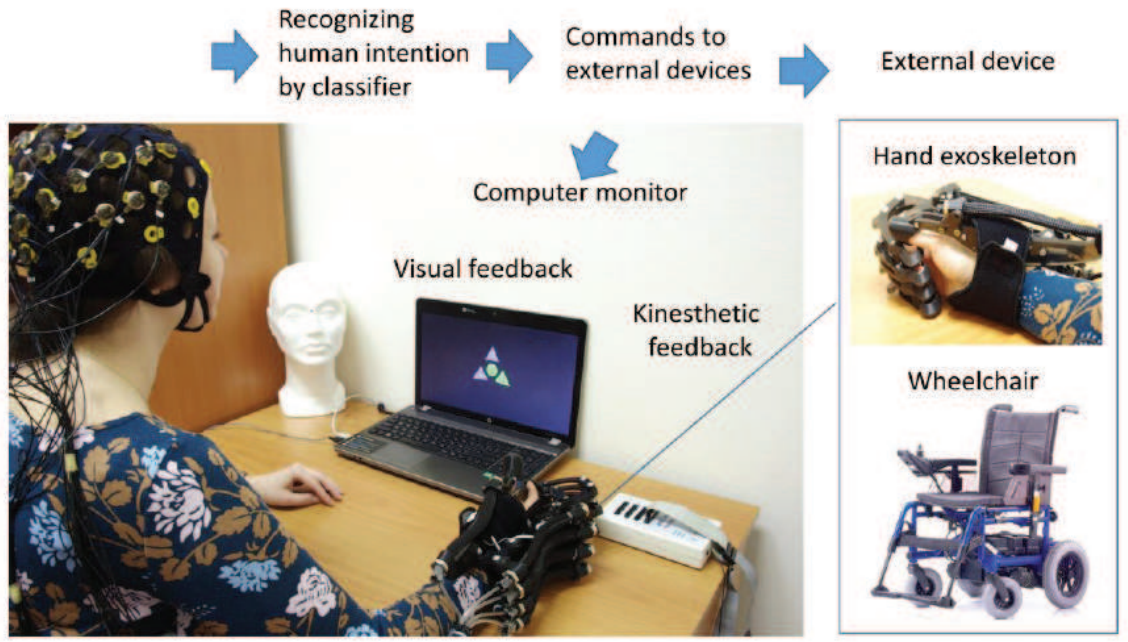

Fig. 1 General scheme of BCI. Brain activity signals are transmitted into computer. With the help of computer processing in real time, classifier recognizes the person's intention. According to the recognition, the command is formed and transmitted to an external device (e.g., computer monitor, exoskeleton or wheelchair).

Despite the fact that the first BCI was established in the early 70s of the last century [89,90], for many years the idea of decoding human thoughts and intentions by the signals of the brain seemed impossible or feasible only in the long-term future because of the high variability and low resolving power of these signals. Furthermore, BCI requires analyzing brain signals in real time, and until recently, the sufficiently powerful technologies were not available or were too expensive. However, over the past twenty years, mostly thanks to new technologies that have come on the market, the situation has radically changed. While twenty years ago in BCI studies only 3 teams of researchers were working actively, then there were 8 such teams ten years ago, and nowadays their number exceeds 100. Accordingly, the number of publications on this topic increases from 10 in the period 1993-95 to 500 between years 2008 and $2010[58,93]$. The largest number of researches relate to the use of EEG because this technology is not invasive and requires cheap and compact equipment.

\subsection{Types of BCI}

In the previous section we classified BCIs according to the type of brain activity measured. On the other hand, they can be classified according to the method of the brain activity signals acquisition and according to the type of their transformation to the external device control command.

According to the method of the brain activity signals acquisition we can classify BCI as follows: The BCI using the brain signals recorded on the surface of 
the scalp (e.g. EEG, NIRS) or outside the head (MEG, fMRI) are non-invasive. The BCI based on the use of the individual neurons activity are invasive since this activity is recorded using microelectrode system that is implanted in the brain. Note that, despite undeniable encouraging results of invasive BCI, their mass-scale use in clinics is still too far. The main obstacles for this are

1) a short time of their effective functioning due to covering the recording electrodes with connective tissue, resulting in the loss of electrical contact with the brain tissue, and

2) the possibility of infection through open cable burr hole.

Therefore, it is expected that non-invasive BCI will be applied most massively in the coming years.

According to the method of brain signals transformation to the external device control command, BCIs can be classified as gradual or discrete. The gradual BCI learns new continuous sensorimotor transformation, linking brain activity with the direction of the desired movement.

A typical experimental protocol with gradual BCI is to ask a subject to shift the computer cursor to an arbitrary target on the screen with the help of mental effort. In the case of non-invasive BCI based on the EEG analysis, the subjects are able after several weeks of training to move the cursor from an arbitrary initial position to an arbitrary target within $2-5$ seconds [94].

However, in the case of invasive BCI, based on the registration of the activity of individual neurons, cursor control is facilitated, as there is direct link between neuronal activity in the motor areas of the cerebral cortex and the desired direction of movement [40]. Namely, each neuron has a preferred direction of movement at which its activity is maximal. In the case of other direction of movement, activity of the neuron is proportional to the cosine of the angle between current direction and the preferred direction. By recording the activity of multiple neurons one can identify their preferred directions in advance, so it is easy to calculate from their current activity the desired direction of movement. The use of gradual invasive BCI, based on the registration of the activity of individual neurons, is typical for experiments on monkeys [63].

The discrete BCI, in contrast to gradual one, is able to send to external technical device only a limited set of commands. It is based on the recognition of spatiotemporal EEG patterns that arise as a response to a given set of carried mental tasks. The idea of this BCI was first proposed in [65]. In most of the studies, the spatial distributions of amplitudes of different EEG rhythms on the surface of the head, which are known to reflect the dominance of certain cognitive processes, are used as spatiotemporal EEG patterns.

The logic of using EEG patterns corresponding to performing different mental tasks is as follows. Initially, the participant is asked to perform several types of mental tasks. Adaptive classifier determines which types of tasks can be classified with the greatest accuracy. Then, in agreement with the participant, each of these tasks is associated with one of the control commands to the external technical device. After this the BCI operator, to execute a desired command, performs the 
proper mental task in the mind. The number of mental tasks and hence the number of available commands to the external device is formally not limited. The main limiting problem is that their respective EEG patterns would be well recognized. If the command for an external device is to perform a certain movement, then it is psychologically convenient to associate the command with some movement imagery. For example, turning the wheelchair to the left is easily associated with the imagery of the left hand movement, and to the right with the right hand movement imagery.

As an example, in Fig. 2 are depicted the results of our experiments with healthy subjects $[17,18,32,34]$ and post-stroke patients $[14,60,66]$ to assess the accuracy of the classification of EEG patterns corresponding to the implementation of three tasks in motor imagery and three tasks in visual imagery. The tasks in the motor imagery were to imagine a flexion of the left or right hand and alternatively to relax. The tasks in the visual imagery were to imagine a house or a chair and alternatively a blank computer screen.

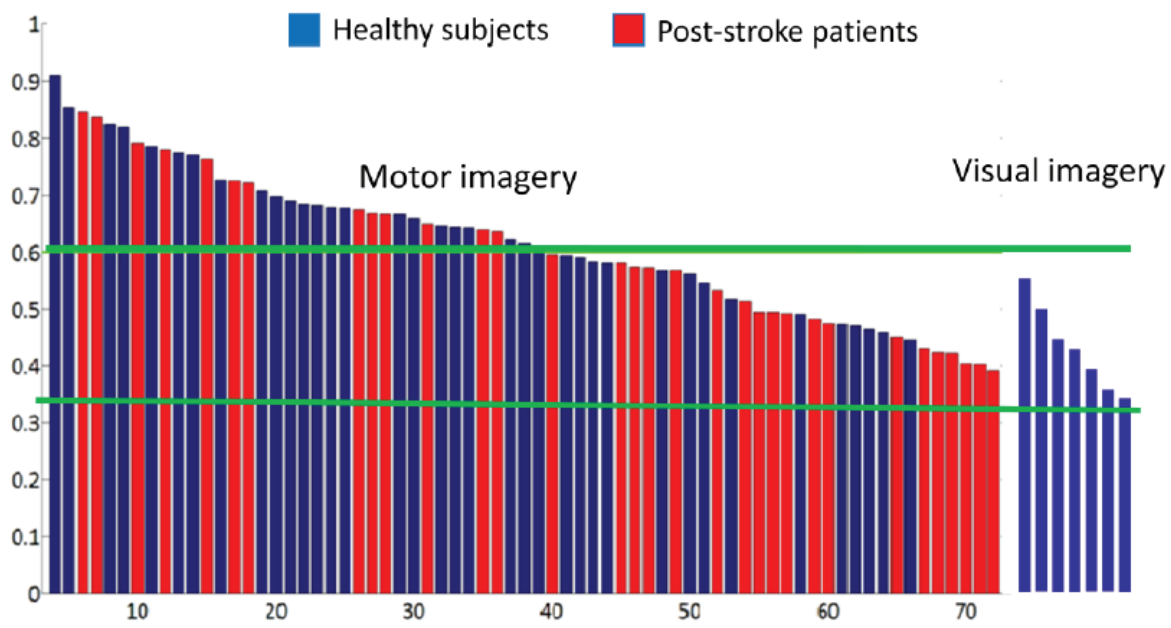

Fig. 2 The proportion of correct classifications of EEG patterns corresponding to performance of three mental tasks in BCI experiments with motor and visual imagery according to [14,17, 18, 32, 34, 60, 66].

The experiments were performed using a standard protocol for this type of research. Each experimental series consisted of four consecutive sessions: preliminary, training and two testing sessions. During preliminary session participants were asked to seat quietly with eyes open and closed, to blink and to move eyes to left, right, up and down. The results of this session were later used to remove oculomotor artifacts and for identification of occipital alpha rhythm. Training and testing sessions served, respectively, for the initial setup of the BCI classifier and its testing. The participants carried out one of the three instructions pointing which mental task must be performed. Instructions were combined into blocks, each containing all instructions in random order. Classifier analyzed EEG patterns over one second window shifting each $250 \mathrm{~ms}$. Classifier response was treated as correct 
if it coincided with the instruction. The details of the experimental protocol are described in [17, 18,32,34].

The participants are ranked in Fig. 2 according to the quality of recognizing their EEG patterns. For the three mental tasks the rate of the accidental recognition is $1 / 3$. The results are shown for the fifth day of training to control BCI. Recognition of EEG patterns was performed by the simple Bayesian classifier based only on comparing covariance matrices of EEG signals corresponding to different mental tasks $[17,18]$. As shown in [31] this classifier only slightly loses to others more sophisticated classifiers significantly winning in computing cost.

As shown in the figure, for both types of tasks, the recognition quality exceeds the random level, but it is much higher in the case of motor imagery. There is a large variability in the quality of recognition between subjects. For about $15 \%$ of participants the proportion of correct recognition during the motor imagery is more than $80 \%$ and for about $20 \%$ of participants this proportion was barely higher than a random level. The similar results were obtained for 324 healthy subjects performing two mental tasks: imagination of left or right hand movement [79]. The quality of EEG pattern recognition is practically independent of classifier $[10,31]$. However, the continuation of the training, as a rule, can significantly increase the recognition quality [46].

Fig. 2 also demonstrates that post-stroke patients are able to perform BCI control as healthy participants. In the frame of the inclusion criteria (see below) we could not find the dependence of recognition quality on location, duration and severity of the brain damage. Thus, the BCI based neurorehabilitation is acceptable for the wide range of post-stroke patients. Since BCI based on the motor imagery is mainly used for motor rehabilitation, we consider only this type of $\mathrm{BCI}$ in the following.

\subsection{Neurophysiological prerequisites for the motor imagery BCIs development}

Movement execution, preparation and even observation are usually accompanied by a decrease in $\mu$ - and $\beta$-rhythm in the cortical area representing the involved body segment. Such a reduction is called event-related desynchronization (ERD) [77,78]. The increase in $\mu$-rhythm, i.e. event-related synchronization (ERS), is observed in the regions of the brain representing body segments, which are not involved in the task [76]. According to M. Jeannerod [51,52] the physiological phenomena accompanying the performance of these tasks are so similar because all of them require an internal movement presentation.

The high efficiency of BCI, based on motor imagery, is due to the fact that the ERD and ERS arise also in the case of kinesthetic motor imagery [76-78] which is the prolonged internal motor presentation $[51,52]$. Kinesthetic motor imagery must be distinguished from visual motor imagery. In the second case, a person imagines that he is seeing his own movement, treating it as if he is observing it from the outside. In the first case it generates kinesthetic filling of the movement. It is shown that kinesthetic motor imagery activates the same brain regions as the real motion, while visual imagery activates mostly visual areas of the brain $[35,68,84]$, so ERD and ERS are reactions to kinesthetic motor imagery. Areas representing 
the sensitivity of the body segments are widely distributed over the surface of the cortex [16]. Therefore, motor imagery of different body segments provides brain activity in different brain areas, consequently, different spatial patterns of EEG. This facilitates the task of recognizing which body segment movement was imagined.

The exact localization of the sources of the electrical brain activity, the most significant for the functioning of BCI, based on the imagination of hand movements, was carried out in $[32,34]$. To find the sources which activity accompanies movement imagination, we used Independent Component Analysis (ICA), which provides representation of a multidimensional EEG signal $\mathbf{X}(t)$ (where components of $\mathbf{X}(t)$ represent electric potentials recorded from $N$ individual electrodes at the head surface) as a superposition of activities of independent components $\xi(t)$ :

$$
\mathbf{X}(t)=\mathbf{W} \xi(t)=\mathbf{W}_{1} \xi_{1}(t)+\mathbf{W}_{2} \xi_{2}(t)+\cdots+\mathbf{W}_{N} \xi_{N}(t) .
$$

Columns $\mathbf{W}_{i}$ of matrix $\mathbf{W}$ specify the contribution of the corresponding independent component (or source) into each of the electrodes and the components $\xi_{i}(t)$ of the vector $\xi(t)$ specify sources activity in each time point. ICA becomes more and more widely used in the multichannel EEG recording analysis, in general, and in the analysis of BCI data, in particular [54]. Combining with the solution of inverse EEG problem [43], ICA promises "to bring EEG once again to the forefront of brain imaging, merging its high time and frequency resolution with enhanced spatial resolution of its cortical sources" [71].

To decompose recorded signals into independent components we used RUNICA, which is a part of the EEGLab package in MATLAB [24]. RUNICA provides selection of independent components by minimizing mutual information between component activities [49]. For each subject on each experimental day we identified three independent components that provide the best recognition of performed mental tasks according Cohen's kappa index [57]. Fig. 3 shows selected components for one of the participants.

The first two components, denoted $\mu 1$ and $\mu 2$, were identified in this participant regularly every experimental day. They demonstrate reaction of both ERD and ERS in response to hand movement imagery. Activity of the component $\mu 1$ is suppressed in the ranges $8-13 \mathrm{~Hz}$ and $20-25 \mathrm{~Hz}$ by the imagining the right hand movement and is enhanced by the imagining the left hand movement. The focus of this component is located in the left hemisphere, i.e. where the sensorimotor areas of the right hand are presented. For the component $\mu 2$, which focus is located in the right hemisphere, ERD and ERS behave reversely as compared with $\mu 1$ : its activity is suppressed by imagining the left hand movement and is enhanced by imagining of the right hand movement. It should be noted that for these independent components, ERD and ERS are much more pronounced than in the case when they are observed directly from recording by individual electrodes. For example, for the participant which data are shown in Fig. 3, the spectral power of the independent components decreased during ERD in the range 8-13 Hz by about 5 times, while recording from the central electrodes $\mathrm{C} 3$ and $\mathrm{C} 4$, where ERD and ERS were expressed most strongly, the spectral power decreased by only $30 \%$. 
Frolov A.A. et al.: Principles of motor recovery in post-stroke...

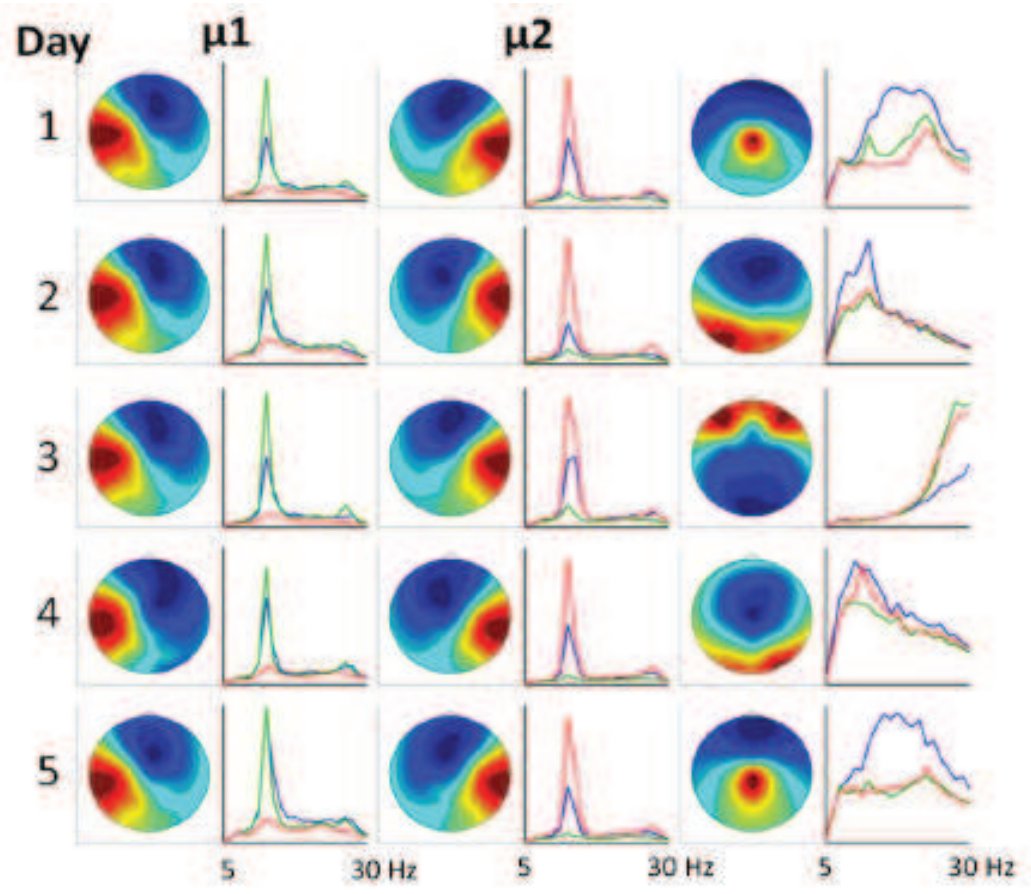

Fig. 3 Triplets of optimal ICA components in each experimental day for one of the participants. Each component is presented as a spatial distribution of its contribution to the electrical potential at the surface of the head and spectral power for activity of each component in different mental states. Spectral power is presented in the range $5-30 \mathrm{~Hz}$. Blue lines - a state of rest, red line - imagining the right hand movement, green lines - imagining the left hand movement.

In contrast to the first two components in triplets shown in Fig. 3 the third component was rather variable and reflected activities of other sources. As an example, Fig. 4 demonstrates 64 topographic maps for the most regularly identified independent components during motor imagery. Components $\mu 1$ and $\mu 2$ can be easily recognized among them. It is reasonable to suppose that independent sources of electrophysiological brain activity are current dipoles distributed over the neocortex [25]. Topographic maps shown in Fig. 4 confirm this assumption: the distribution of electrical potential over the head produced by each of these sources could be actually interpreted in terms of electrical field produced by a single current dipole.

According to fMRI study, about 35 brain areas are activated during motor imagery $[44,47]$. The number of sources of electrophysiological activity revealed by ICA is even higher but not all of them are specific to motor imagery. Explaining the functional meaning of the revealed sources is a task for future study.

Sources of electrical activity $\mu 1$ and $\mu 2$, which happened to be the most relevant for BCI performance, have been localized by solving the inverse EEG problem in a single dipole approximation $[19,32,34]$. In other words, position and orienta- 


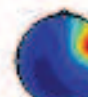

8

,
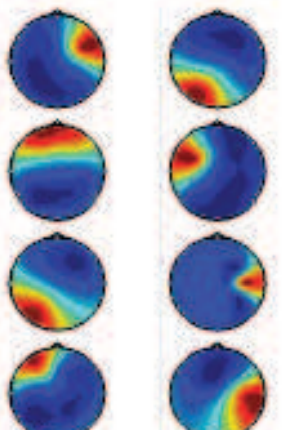

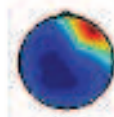
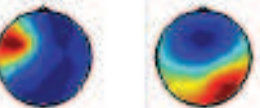

.
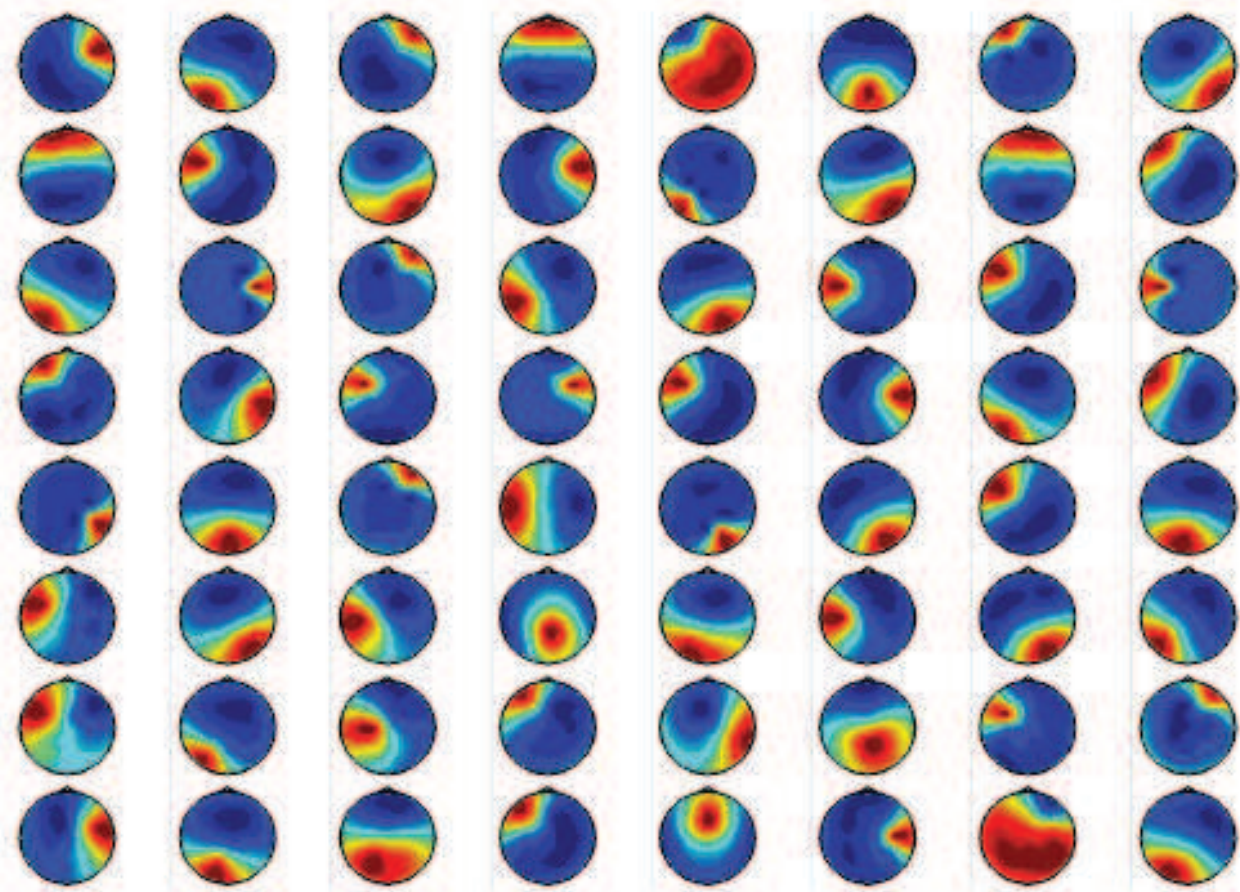

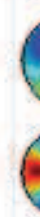
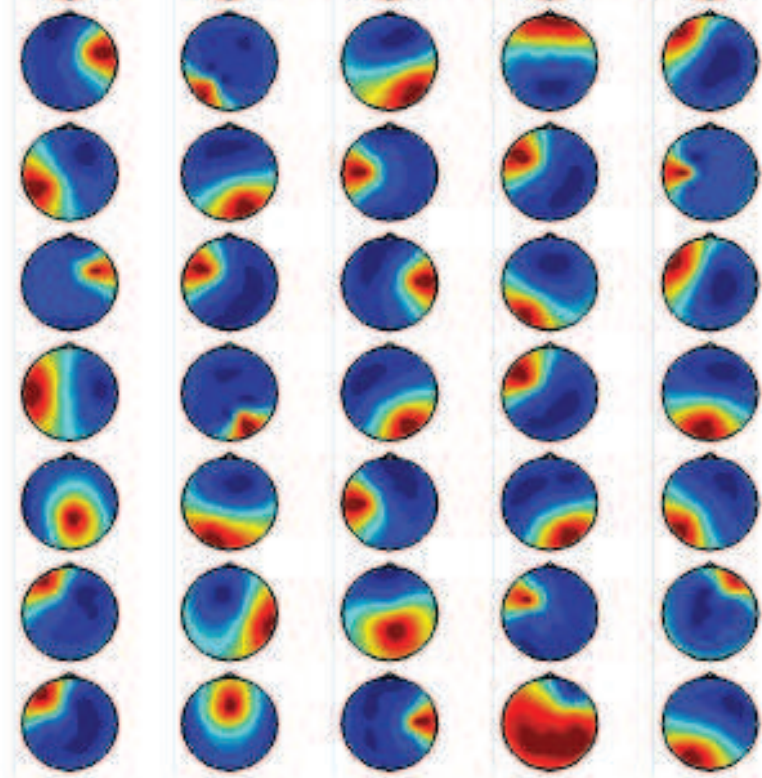

Fig. 4 Topographic maps of independent components most often revealed by ICA during motor imagery.

tion of a single current dipole were searched to match maximally patterns of EEG distribution on the head surface given by ICA and by a dipole. The pattern of EEG distribution for dipole with a given position and orientation was calculated by solving the direct EEG problem. It was solved by the finite element method (FEM) which allows to take into account individual geometry of the brain and its covers. In FEM, one critical requirement for the mesh generation is proper representation of the geometric and electrical properties of the head volume conductors. To generate the FEM meshes from the MRI data, MR images were segmented into five sub-regions: white matter, gray matter, cerebrospinal fluid, skull and scalp. To construct the FE models of the whole head the FEM mesh generation was performed using tetrahedral elements with inner-node spacing of $2 \mathrm{~mm}$. Thus, the total number of nodes amounted to about 1.5 millions. To solve the direct EEG problem, the FEM mesh along with electrical conductivities was imported into the commercial software ANSYS (ANSYS, Inc., PA, USA).

If direct EEG problem is solved taking into account the individual geometry of the brain and its coverings the mean coordinates of source $\mu 1$ in the standard head model presented in MNI (Montreal Neurologic Institute) brain atlas amount $(-32.5 \mathrm{~mm},-24.7 \mathrm{~mm}, 54.0 \mathrm{~mm})$. The individual coordinates were scattered in the sphere of radius $5 \mathrm{~mm}$. The mean coordinates correspond to source position in Brodmann area $3 \mathrm{a}$ in the depths of the central sulcus which is responsible for the 
proprioceptive sensitivity of the hand and fingers [55]. If direct EEG problem is solved using standard head model the mean source coordinates amount $(-29.4 \mathrm{~mm}$, $-13.9 \mathrm{~mm}, 59.6 \mathrm{~mm}$ ) and individual coordinated are scattered in the sphere of radius $15 \mathrm{~mm}$, i.e. much larger than in the case when individual head geometry is taken into account. The mean sources coordinates are higher and more anterior and correspond to premotor Brodmann area 6. Similar results were obtained for $\mu 2$.

Localization of the sources $\mu 1$ and $\mu 2$ in the primary somatosensory area corresponds to the common notion that rhythms of alpha range are rhythms of the primary sensory areas. This is also consistent with the localization of the murhythm sources obtained by MEG in humans [53] and by ECoG in cats [82]. However, in [67] they were localized by EEG in the precentral motor area. We suppose that this localization is not correct because it was carried out without taking into account the individual geometry of the brain and its coverings.

Thus, ERD and ERS, i.e. suppression of mu-rhythm in the area responsible for proprioceptive sensitivity of the body segment whose movement is imagined and its enhancement in other brain areas, are the main neurophysiological prerequisites of successful BCI operation.

\section{Movement control of human arm and exoskeleton}

\subsection{Proportional derivative controller}

Within the BCI paradigm, signals of the brain activity associated with the imagined movement are processed in real time in order to form the proper command to exoskeleton moving the arm. Comfortable interaction between exoskeleton and the human arm is achieved when movements of the exoskeleton are controlled according to the same principles as human movements. Otherwise the movement produced by the exoskeleton are perceived by human as imposed and not corresponding to his intention. In this Section we describe some biologically adequate principles to control exoskeletons used in our experiments [33].

For goal-directed arm movement, the human intention can be formalized in the form of the working point (WP) trajectory, such as the hand trajectory to capture an object or the trajectory of the fingertip for getting into a point. Problem of transforming the working point trajectory into rotations in the joints has no unique solution due to the kinematic redundancy of the human arm. This transformation is learnt due to motor practice and one of the possible neural network models of such learning is given in [28]. The result of the transformation of the working point into the desired changes in joint angles is strictly individual and is related rather to the individual biomechanical properties than to the difference in control [45]. In our control system, the desired WP trajectory is transformed into the desired trajectory in the space of joint angles by transforming the WP velocity $\mathbf{V}_{W P}$ into the joint angular velocity $\mathbf{V}_{J A}$ as

$$
\mathbf{V}_{J A}=\mathbf{J}^{+} \mathbf{V}_{W P},
$$

where $\mathbf{J}$ is the Jacobian of transformation of joint angles into WP position. 
It is shown in the numerous experimental physiological studies (for example, $[15,29,30,41,74])$, that when the desired joint angle trajectory $\varphi^{\operatorname{des}}(t)$ is given it is transformed into total joint muscle torques by a PD (Proportional Derivative) controller

$$
\mathbf{T}(t)=-\mathbf{T}^{\mathrm{gr}}(\varphi(t-\tau))+\mathbf{S}\left(\varphi^{\mathrm{des}}(t)-\varphi(t-\tau)\right)-\mathbf{V} \dot{\varphi}(t-\tau),
$$

where $\varphi$ is a vector of joint angles, $\tau$ is some time delay and $-\mathbf{T}^{\text {gr }}$ is a vector of static muscle torques designed to compensate gravity forces. PD - controller is equivalent to visco-elastic spring with adjustable parameters: "equilibrium point" $\varphi^{\text {des }}$, "stiffness" $\mathbf{S}$ and "viscosity" $\mathbf{V}$.

Stiffness and viscosity of the joints during goal-directed movements are determined experimentally using short random external perturbations applied to the moving body segments. It is reasonable to assume that the central nervous system does not have time to react to these perturbations, and therefore, $\varphi^{\text {des }}(t)$ remains to be the same in perturbed and unperturbed movements. Then stiffness, viscosity and time delay can be calculated using a linear regression model relating the change in joint torques $\Delta \mathbf{T}=\mathbf{T}_{\text {up }}-\mathbf{T}_{\mathrm{p}}$ with the change in joint angles $\Delta \varphi=\varphi_{\text {up }}-\varphi_{\mathrm{p}}$ and angular velocities $\boldsymbol{\Delta} \dot{\varphi}=\dot{\varphi}_{\text {up }}-\dot{\varphi}_{\text {p }}$ caused by disturbance (e.g, [5, 6, 30, 42]):

$$
\Delta \mathbf{T}(t)=-\Delta \mathbf{T}^{\mathrm{gr}}(t-\tau)-\mathbf{S} \boldsymbol{\Delta} \varphi(t-\tau)-\mathbf{V} \boldsymbol{\Delta} \dot{\varphi}(t-\tau),
$$

where $\Delta \mathbf{T}^{\mathrm{gr}}=\mathbf{T}^{\mathrm{gr}}\left(\varphi_{\mathrm{up}}(t-\tau)\right)-\mathbf{T}^{\mathrm{gr}}\left(\varphi_{\mathrm{p}}(t-\tau)\right)$ and indices "up" and "p" relates to unperturbed and perturbed movements.

To use this method, one must record the kinematics of motion and calculate the total joint torques produced by muscle forces from Lagrangian dynamic equation:

$$
\mathbf{I}(\varphi) \ddot{\varphi}+\mathbf{C}(\varphi) \times \dot{\varphi} \times \dot{\varphi}=\mathbf{T}^{\mathrm{gr}}+\mathbf{T}+\mathbf{J}_{\mathrm{ext}}^{\mathrm{T}} \mathbf{F}_{\mathrm{ext}},
$$

where $\varphi$ is a vector of joint angles, $\mathbf{I}$ is a matrix of inertia, $\mathbf{T}^{\mathrm{gr}}=-\operatorname{grad}_{\varphi} P$ is a vector of torques produced by gravity forces $(P$ is a body potential energy in the gravitational field), $\mathbf{T}$ is a vector of joint torques produced by muscle forces, $\mathbf{F}_{\text {ext }}$ is a vector of external forces producing perturbation, $\mathbf{J}_{\text {ext }}$ is a Jacobian defining the relation between the points of external force application $\mathbf{r}_{\text {ext }}$ and joint angles by equation $\dot{r}_{\text {ext }}=\mathbf{J}_{\text {ext }} \dot{\varphi}, \mathbf{C}$ is a tensor defining Coriolis and centripetal forces, so that $(\mathbf{C}(\varphi) \times \dot{\varphi} \times \dot{\varphi})_{i}=\sum_{j, k} \mathbf{C}_{i, j, k} \dot{\varphi}_{j} \dot{\varphi}_{k}$. A convenient way how to calculate matrix $\mathbf{I}$ and tensor $\mathbf{C}$ one can find in [59]. Torques produced by muscle forces are calculated by Eq. (5) when the movement is unperturbed $\left(\mathbf{F}_{\text {ext }}=0\right)$ and when it is perturbed. In order to record the movement kinematics used are goniometers [45], optical [48] or electromagnetic [7] systems. In our studies recordings were taken by using electromagnetic spatial tracking systems (Fastrack Polhemus), Flock of Birds, MiniBirds, TrakSTAR (Ascension Technology Corp.). To record movement kinematics, the sensors are mounted at the body segments. During the movement, recorded are coordinates and orientation of each sensor relative to the stationary coordinate system associated with an electromagnetic field transmitter. Thus, the motion of each sensor is described by 6 parameters $(3$ coordinates and 3 Euler angles). Such a description is redundant: for example, to describe the human arm with 7-degrees of freedom (two in the wrist, two in the elbow and three in the shoulder joint), $24(6 \times 4)$ parameters are recorded from 4 sensors mounted at the 
Frolov A.A. et al.: Principles of motor recovery in post-stroke...

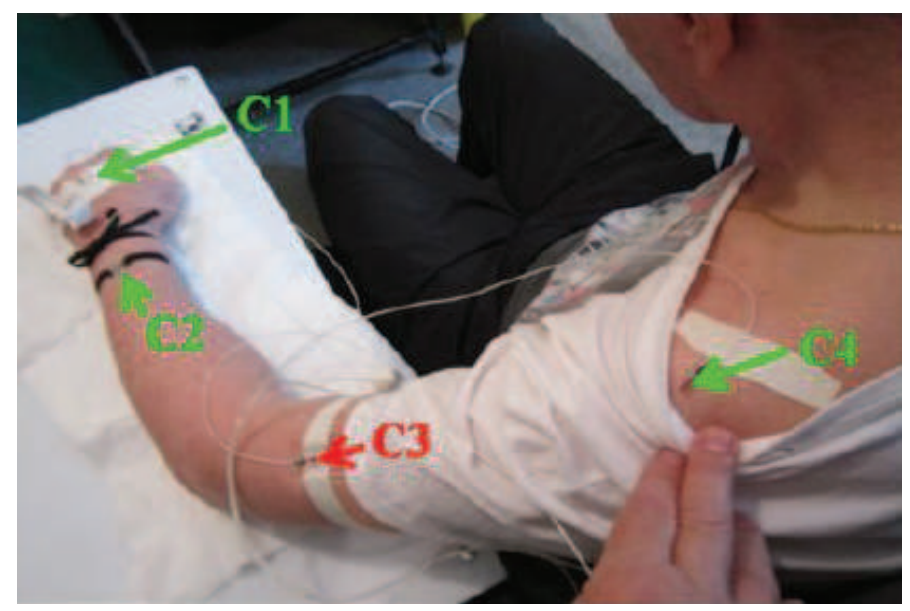

Fig. 5 Recording of arm movements by 4 sensors of the MiniBirds electromagnetic system fixed to the hand (C1), forearm (C2), upper arm (C3), and scapula (C4).

hand, forearm, upper arm and scapula (Fig. 5). This allows for using different optimization methods for calculating the positions and orientations of the axes of rotations in the joints as well as angles of rotations around these axes during the arm movement $[13,80]$.

In humans, stiffness and viscosity of the spring are determined by the interaction of three different physiological mechanisms: by visco-elastic properties of muscle fibers and tendons, spinal stretch reflex loop and the feedback loop passing through the brain [74]. In contrast to the first mechanism, second and third loops have significant delays. For the stretch reflex loop, this delay amounts to about $50 \mathrm{~ms}$, and for the second loop - up to $150 \mathrm{~ms}[29,30,74]$. The effective delay used in the $\mathrm{PD}$ controller is determined by the relative contributions of all these three mechanisms. The presence of a time delay imposes restrictions on the feedback parameters to ensure a stable control: the values of stiffness and viscosity should be bounded both from above and from below [5]. For a variety of published data stiffness amounts to the range $2-25 \mathrm{Nm} / \mathrm{rad}$ at the elbow and $10-35 \mathrm{Nm} / \mathrm{rad}$ in the shoulder joint (e.g. [30]), that is orders less than those used in the industrial robots. Namely this makes the human movements smooth and flexible to the external perturbations. However, under the relatively small values of the feedback gain parameters, the biomechanical system obtains the resonance properties with the frequencies close to the frequencies of natural movements. This can result in the resonance oscillations of high amplitude. The visco-elastic nature of the joint torque under conditions of low values of joint stiffness has been formulated by N.A. Bernstein as one of the main difficulties of motor control by CNS [11]. To overcome, this problem the PD-control needs to take into account not only the kinematics of a given joint, but also those of all other joints. In this case, matrices of stiffness $\mathbf{S}$ and viscosity V in Eq. (3) are not diagonal. Such a kind of control is called "fullstate feedback control" [9,73]. Moreover, the special selection of the feedback loop parameters is required to avoid instability and resonance oscillations. The most 
common approach to find such parameters is the LQR method (Linear Quadratic Regulation, [86]). It cannot be used, however, in the presence of delays. As shown in [3-6] this problem is overcome in humans by using of independent control of special motor synergies described in the next Section.

The adequacy of PD - controller with time delay, described by Eq. (3), as a model of controlling total joint torques produced by muscular forces has been shown for the case of controlling the elbow during unexpected and controlled forearm unloading [12], human arm reaching movements [30] and human bending [5,6] and sway [75] in the sagittal plane. However, there exist many more sophisticated models of movement control in human, e.g. the model which claims that joint torques required to execute a desired movement are directly calculated by CNS (e.g. [56]) or the model which claims that time delays inherent for CNS are overcome by prediction of future kinematics [39].

\subsection{Motor synergies}

In the process of motor learning so-called motor synergies are formed - coordinated motion in the limb joints controlled by a single command [11]. Motor synergies manifest themselves in the synchronized changes in the joint angles (kinematic synergies) or the joints torques (the dynamic synergy). The relationship between the kinematic and dynamic synergies is determined by a complex dynamic interaction between segments of a biomechanical chain. The movement of each segment has an effect on the motion of all the others, and any muscle activation involves movement of all segments, not only those in which this muscle is attached. As a consequence, the simplicity of the kinematic pattern does not guarantee the simplicity of dynamic pattern or the simplicity of control commands.

Controlling multi-joint biomechanical system would be much easier if there were such movement classes, for which the kinematic synergy would occur simultaneously with the dynamic one. Such movements could constitute a library of motor control modules. As was shown by studying human bending in sagittal plane, $[3,4]$ the movements along the eigenvectors of the linearized dynamic equation satisfy this condition: for them dynamic synergy is accompanied by kinematic synergy.

Dynamic Eq. (5) in the vicinity of some equilibrium position $\varphi_{0}$ can be represented in the linear approximation as

$$
\mathbf{I}_{0} \ddot{\varphi}_{1}-\mathbf{D}_{0} \varphi_{1}=\mathbf{T}_{1},
$$

where $\mathbf{I}_{0}=\mathbf{I}\left(\varphi_{0}\right), \varphi_{1}=\varphi-\varphi_{0}, \mathbf{T}_{1}=\mathbf{T}-\mathbf{T}_{0}$ and matrix $\mathbf{D}_{0}$ defines gravitational forces as $\mathbf{T}^{\mathrm{gr}}(\varphi)=\mathbf{T}^{\mathrm{gr}}\left(\varphi_{0}\right)+\mathbf{D}_{0} \varphi_{1}$.

In this case, the multi-joint movement can be represented as a superposition of "eigenmovements" which are the movements along the eigenvectors of Eq. (6). By definition each eigenvector $\mathbf{W}_{i}$ satisfies equation

$$
\mathbf{I}_{0} \mathbf{W}_{i}=\lambda_{i} \mathbf{D}_{0} \mathbf{W}_{i},
$$

where $\lambda_{i}$ is a corresponding eigenvalue. Presenting $\varphi_{1}=\mathbf{W} \xi$ and $\mathbf{T}_{1}=\mathbf{D}_{0} \mathbf{W} \eta$ we obtain the dynamic equation in the form:

$$
\Lambda \ddot{\xi}-\xi=\eta,
$$


Frolov A.A. et al.: Principles of motor recovery in post-stroke...

where $\boldsymbol{\Lambda}$ is a diagonal matrix of eigenvalues. In terms of eigenmovements PDcontroller given by Eq. (3) takes the form:

$$
\eta(t)=-\xi(t-\tau)+\mathbf{S}^{\text {eig }}\left(\xi^{\text {des }}(t-\tau)-\xi(t-\tau)\right)-\mathbf{V}^{\text {eig }} \dot{\xi}(t-\tau),
$$

where

$$
\mathbf{S}^{\text {eig }}=\left(\mathbf{D}_{0} \mathbf{W}\right)^{-1} \mathbf{S W} \text { and } \mathbf{V}^{\text {eig }}=\left(\mathbf{D}_{0} \mathbf{W}\right)^{-1} \mathbf{V W}
$$

are stiffness and viscosity matrices in terms of eigenmovement. The linked system of dynamic equations Eq. (6) splits into independent dynamic equations if $\mathbf{S}^{\text {eig }}$ and $\mathbf{V}^{\text {eig }}$ are diagonal matrices, that is, in the case when eigenmovements are controlled independently. As shown in [4-6], this condition satisfies at least for human bending. We generalized this suggestion for any arbitrary movement [33].

Independent control of eigenmovements allows for calculating parameters of the PD-controller to avoid instability and resonance oscillations even when this controller has a time delay. Dynamic Eq. (7) when the vector of generalized torques $\eta$ satisfies Eq. (8) and matrices $\mathbf{S}^{\text {eig }}$ and $\mathbf{V}^{\text {eig }}$ are diagonal take the form:

$$
\lambda_{i} \ddot{\xi}_{i}(t)-\xi_{i}(t)=-\xi_{i}(t-\tau)+\mathbf{S}_{i}^{\text {eig }}\left[\xi_{i}^{\text {des }}(t-\tau)-\xi_{i}(t-\tau)\right]-\mathbf{V}_{i}^{\text {eig }} \dot{\xi}_{i}(t-\tau) .
$$

Eq. (10) defines the movement of the pendulum: if $\lambda_{i}>0$ it is inverted, if $\lambda_{i}<0$ it is not inverted.

The stability of each eigenmovement is defined by the roots $\mu$ of secular equation of Eq. (10):

$$
\mu^{2} \lambda_{i}-1+\left(S_{i}^{\mathrm{eig}}+1\right) e^{-\mu \tau}+\mu V_{i}^{\mathrm{eig}} e^{-\mu \tau}=0 .
$$

As shown in [5], when $\tau>0$, Eq. (11) has an infinite number of complex roots $\mu=$ $\alpha+\mathrm{i} \omega$, where $\alpha$ and $\omega$ are real and imaginary parts of the root and $\mathrm{i}$ is the imaginary unit. The solution of Eq. (10) is stable if the real part $\alpha$ of all the roots of Eq. (11) is negative. The maximum value of the real part of all the roots of the secular equation is called the Lyapunov index.

Thus, the solution of Eq. (10) is stable if its Lyapunov index $\alpha<0$. The Lyapunov index defines the characteristic time $\tau_{\mathrm{chr}}=|\alpha|^{-1}$ for the complex system to response to the external perturbation.

The minimization of the Lyapunov index for each eigenmovement was used as a criterion for optimizing PD-controller parameters. The optimum parameters were obtained according to the procedure based on calculation of range in the plane $S_{i}^{\text {eig }} \times V_{i}^{\text {eig }}$ in which the Lyapunov index does not exceed given value $\alpha$. This range will be called $\alpha$-range. For each $\alpha$, the range is defined by the crosscut of two branches of solution of Eq. (11). One branch corresponds to the real roots $\mu=\alpha$. In this case, $S_{i}^{\text {eig }}$ and $V_{i}^{\text {eig }}$ satisfy equation:

$$
1+S_{i}{ }^{\text {eig }}+\alpha V_{i}^{\text {eig }}+\left(\lambda_{i} \alpha^{2}-1\right) e^{\alpha \tau}=0,
$$

which corresponds to the straight-line in the $\left(S_{i}{ }^{\text {eig }} \times V_{i}^{\text {eig }}\right)-$ plane. The second branch corresponds to the roots $\mu$ with non-zero imaginary parts. Then the values $S_{i}^{\text {eig }}$ and $V_{i}^{\text {eig }}$ for a given root $\mu=\alpha+\mathrm{i} \omega$ can be found as the solution of the system of two real equations

$$
S_{i}^{e i g} \cos (\omega \tau)+V_{i}^{e i g}[\alpha \cos (\omega \tau)+\omega \sin (\omega \tau)]=\left[1+\lambda\left(\omega^{2}-\alpha^{2}\right)\right] e^{\alpha \tau}-\cos (\omega \tau),
$$




$$
S_{i}^{e i g} \sin (\omega \tau) / \omega+V_{i}^{e i g}[\alpha \sin (\omega \tau) / \omega-\cos (\omega \tau)]=2 \lambda \alpha e^{\alpha \tau}-\sin (\omega \tau) / \omega .
$$

In Fig. 6 are depicted three examples of $\alpha$-ranges $\left(\alpha=0, \alpha=-2.0 \mathrm{~s}^{-1}\right.$ and $\left.\alpha=-4.0 \mathrm{~s}^{-1}\right)$ for $\lambda_{i}=0.1 \mathrm{~s}^{2}$ and $\tau=0.1 \mathrm{~s}$. Each $\alpha$-range is confined by two lines. The lower line for each range gives the solution of Eq. (12) and the upper line gives the solution for the system of Eqs. (13) and (14) with $\omega$ as parameter. The more negative $\alpha$ is, the narrower the $\alpha$-range becomes. Within the $\alpha$-range for $\alpha=0$ the real parts of all the roots of Eq. (11) are negative and the system is stable. Outside this range, the system is unstable. For given values of $\lambda_{i}$ and $\tau$, there exists a minimum value $\alpha_{\min }$ where the $\alpha$-range disappears. The open circle in Fig. 6 indicates the corresponding point. This point defines the optimal parameters $S_{i}^{\text {eig }}$ and $V_{i}^{\text {eig }}$ of the PD-controller feedback loop that provide the fastest possible response of the system to external perturbation and the minimum delay in the transformation of the desired kinematics to the actual one. For $\lambda_{i}=0.1 \mathrm{~s}^{2}$ and $\tau=0.1 \mathrm{~s}$ optimal parameters amount to $S_{i}^{\text {eig }}=0.7$ and $V_{i}^{\text {eig }}=0.5 \mathrm{~s}$ and $\alpha_{\min }=-5 \mathrm{~s}^{-1}$, that is characteristic time to response to external perturbation amounts up to $200 \mathrm{~ms}$. It is worth noting that the root of Eq. (11) corresponding to this minimal Lyapunov index has no imaginary part. Thus, this response is not oscillatory. All roots containing imaginary parts have much more negative real parts comparing to $\alpha_{\min }$, so the probability of resonance oscillations is small.

The inverse transformation of Eq. (9) gives the stiffness and viscosity matrices $\mathbf{S}$ and $\mathbf{V}$ in Eq. (4) in terms of joint angles:

$$
\mathbf{S}=\mathbf{G}_{0} \mathbf{W} \mathbf{S}^{\mathrm{eig}} \mathbf{W}^{-1} \mathbf{V}=\mathbf{G}_{0} \mathbf{W} \mathbf{V}^{\mathrm{eig}} \mathbf{W}^{-1} .
$$

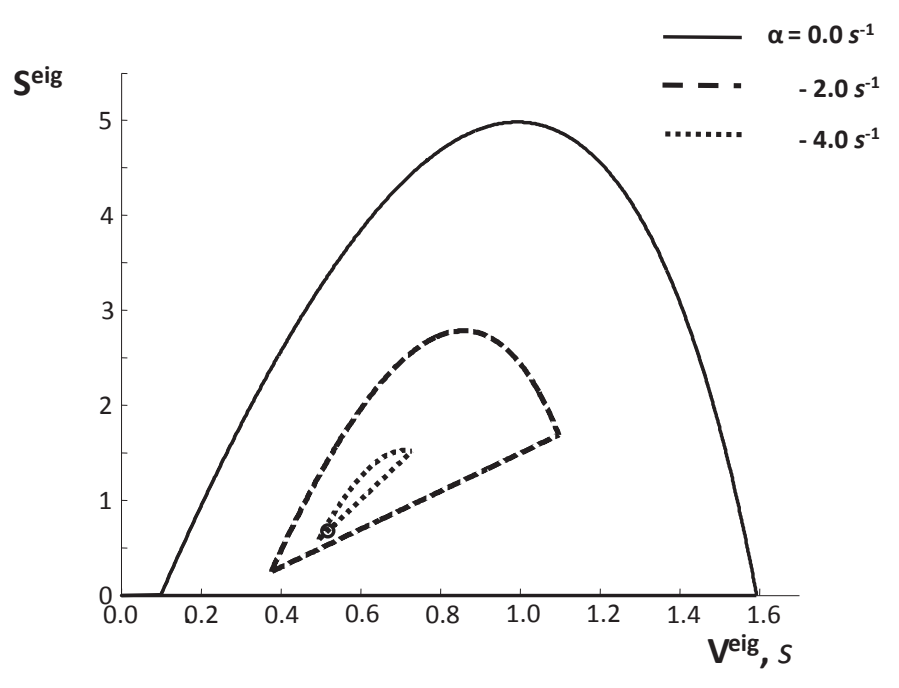

Fig. 6 Dependency of $\alpha$-range on $\alpha$ for $\lambda_{i}=0.1 \mathrm{~s}^{2}, \tau=0.1 \mathrm{~s}$. The open circle marks the optimal values $S_{i}^{\mathrm{eig}}$ and $V_{i}^{\mathrm{eig}}$ that provide the fastest possible response of the system to the external perturbation. 
It has been shown [5] that when matrices $\mathbf{S}^{\text {eig }}$ and $\mathbf{V}^{\text {eig }}$ are diagonal, then matrices $\mathbf{S}$ and $\mathbf{V}$ are symmetrical, but non-diagonal. Therefore, the independent control in terms of eigenmovements corresponds to full-state control in terms of joint angles.

The calculation of eigenvectors was carried out for the biomechanical model of the "standard" human arm, including seven its basic degrees of mobility: three at the shoulder joint ( 1 - flexion-extension, 2 - abduction-adduction, and 3 -rotation about the longitudinal axis), two at the elbow joint (4 - flexion-extension of the forearm with respect to the upper arm and 5 pronation - supination of the forearm with respect to the longitudinal axis) and two at the wrist (6 - flexion-extension and 7 - abduction-adduction). Mechanical parameters of the 'standard' arm were taken from [92].

\begin{tabular}{llrrrrrrr}
\hline Eigenvectors & & $\mathbf{W}_{1}$ & \multicolumn{1}{c}{$\mathbf{W}_{2}$} & \multicolumn{1}{c}{$\mathbf{W}_{3}$} & $\mathbf{W}_{4}$ & \multicolumn{1}{c}{$\mathbf{W}_{5}$} & \multicolumn{1}{c}{$\mathbf{W}_{6}$} & $\mathbf{W}_{7}$ \\
\hline \multirow{3}{*}{ Shoulder joint } & F-Es & 0.00 & 0.02 & 0.08 & -0.01 & -0.10 & $\mathbf{0 . 3 2}$ & 0.11 \\
& Ab-Ads & -0.03 & 0.25 & -0.06 & -0.03 & -0.05 & -0.13 & $\mathbf{0 . 3 8}$ \\
& Rots & 0.05 & $-\mathbf{0 . 3 9}$ & 0.19 & -0.10 & 0.00 & -0.07 & 0.10 \\
\hline \multirow{2}{*}{ Elbow joint } & F-Es & -0.04 & 0.13 & $\mathbf{0 . 6 1}$ & -0.11 & 0.04 & -0.26 & -0.09 \\
& P-Se & -0.24 & -0.21 & 0.14 & $-\mathbf{0 . 8 5}$ & -0.14 & -0.07 & $-\mathbf{0 . 3 7}$ \\
\hline \multirow{2}{*}{ Wrist } & F-Ew & $-\mathbf{0 . 9 7}$ & $-\mathbf{0 . 7 9}$ & 0.11 & $\mathbf{0 . 4 9}$ & -0.22 & -0.21 & 0.14 \\
& Ab-Adw & 0.06 & $\mathbf{0 . 3 3}$ & $\mathbf{0 . 7 4}$ & -0.06 & $\mathbf{0 . 9 6}$ & $\mathbf{0 . 8 7}$ & $\mathbf{0 . 8 2}$ \\
\hline Eigenvalues, $s^{2}$ & & 0.009 & 0.019 & 0.033 & -0.009 & -0.014 & -0.031 & -0.020 \\
\hline \multirow{2}{*}{$\mathbf{S}^{\text {eig }}$} & & 14.5 & 16.7 & 17.5 & 22.7 & 21.4 & 19.9 & 20.5 \\
\hline $\mathbf{V}^{\text {eig }}, s$ & & 11.7 & 9.5 & 8.7 & 3.5 & 4.8 & 6.3 & 5.7 \\
\hline
\end{tabular}

Tab. I The eigenvectors of the linearized dynamic equation for the standard human arm.

The eigenvectors of the Lagrangian dynamic equation of the standard human arm are shown in Tab. I. The dynamic equation was linearized at the point $\left(\varphi_{1}=0, \varphi_{2}=1.2, \varphi_{3}=0, \varphi_{4}=2, \varphi_{5}=0, \varphi_{6}=0.5, \varphi_{7}=0\right.$, where joint angles are given in radians. Zero joint angles correspond to the vertical arm directed downward. Numbering of joint angles correspond to numbering of degrees of mobility. The point of linearization corresponds to hand position in front of the chest at the distance of $20 \mathrm{~cm}$. Columns in Tab. I define the contribution of joint angles into each eigenvector. In some of the eigenvectors only one of degree of mobility dominates $\left(W_{1}, W_{5}\right)$, others are more complex. Three first eigenvalues are positive. It means that the movement along these eigenvectors corresponds to the movement of the inverted pendulum. Other eigenvalues are negative. This corresponds to the movement of not inverted pendulum. The sign of eigenvalue depends on the arm position and configuration. In the point of linearization the forearm and the hand are directed upward and they create a double inverted pendulum. Since flexion-extension in the elbow and movements in the wrist most contribute into the first three eigenvectors, then the first three eigenvalues are negative. Upper arm is 
directed downward, thus it creates not inverted pendulum and the two last eigenvalues are negative since the movements in the shoulder joints mainly contribute to the corresponding eigenvectors. However, generally the sign of the eigenvalue depends on the contribution of all degrees of mobility into the eigenvector and the fifth eigenvalue is negative although the contribution of the wrist abduction adduction into the corresponding eigenvector is large.

For each eigenmovement, the values $S_{\mathrm{i}}^{\text {eig }}$ and $V_{\mathrm{i}}^{\text {eig }}$ were calculated for $\tau=50 \mathrm{~ms}$ and $\alpha=-6 s^{-1}$ so that the corresponding root of the secular Eq. (11) would be real, that is $S_{\mathrm{i}}^{\text {eig }}$ and $V_{\mathrm{i}}^{\text {eig }}$ satisfy Eq. (12). The corresponding values $S_{\mathrm{i}}^{\text {eig }}$ and $V_{\mathrm{i}}^{\text {eig }}$ are also shown in Tab. I. For $\alpha=-6$ the movement is stable and characteristic time to response to external perturbation amounts to $160 \mathrm{~ms}$.

Matrices $\mathbf{S}$ and $\mathbf{V}$ in terms of joint angles obtained by (15) are

$$
\mathbf{S}=\left(\begin{array}{rrrrrrr}
4.4 & -0.8 & -0.2 & 1.2 & -0.2 & 0.0 & -0.2 \\
-0.8 & 2.3 & 0.9 & -0.4 & -0.1 & 0.0 & 0.0 \\
-0.2 & 0.9 & 1.8 & 0.1 & -0.2 & 0.1 & 0.0 \\
1.2 & -0.4 & 0.1 & 1.9 & -0.3 & 0.0 & -0.1 \\
-0.2 & -0.1 & -0.2 & -0.3 & 0.1 & 0.0 & 0.0 \\
0.0 & 0.0 & 0.1 & 0.0 & 0.0 & 0.0 & 0.0 \\
-0.2 & 0.0 & 0.0 & -0.1 & 0.0 & 0.1 & 0.1
\end{array}\right) \mathrm{Nm}
$$

Their components are in the range of experimental values [30].

When moving along some trajectory the system can lose stability if it becomes far from the equilibrium position $\varphi_{0}$, in which vicinity the dynamic equation was linearized. To avoid this loss, matrices $\mathbf{S}$ and $\mathbf{V}$ have to be recalculated according to (15) several times along the trajectory.

The reaching movements calculated with and without perturbation by solving dynamic Eq. (5) with total torques given by PD-controller (4) whose parameters are given by Eq. (15) are shown in $[36,37]$. The same movement produced by the skeleton controlled by PD-controller with parameters obtained by the eigenmovement approach described above is shown in [38]. The exoskeleton was manufactured by the company "Android Technique", Russia. Mechanical parameters of each segment of the combined system of human arm and exoskeleton were calculated for "standard human" and data provided by the company. After calculating desired joint torques $\mathbf{T}^{\text {des }}$ according to the described eigenmovement approach the signal of pulse width modulation $\mathbf{P}$ controlling the current joint torques $\mathbf{T}$ was calculated by the IP (integral proportional) controller as

$$
\dot{P}=\mathbf{K}_{\mathrm{I}}\left(\mathbf{T}^{\text {des }}-\mathbf{T}\right)+\mathbf{K}_{\mathrm{P}}\left(\dot{\mathbf{T}}^{\text {des }}-\dot{\mathbf{T}}\right) .
$$




\section{Clinical Application of Exoskeleton Controlled by BCI}

\subsection{General Patient Characteristic}

The blind, randomized, controlled study has been conducted since December 2014 at three medical centers in Moscow, Russia. The study protocol was registered in the system clinicaltrials.gov: NCT02325947. The study will continue until 120 patients will have completed their whole rehabilitation course.

The inclusion criteria were as follows: male or female patients aged from 18 to 80 years, with the post-stroke period of 1 month to 2 years; hand paresis, mild to plegia, according to the Medical Research Council Sum-Score scale [22]; a single focus of ischemic or hemorrhagic stroke with the supratentorial localization (according to MRI or CT data); a signed informed consent. Patients could be treated at the medical centers or could receive outpatient treatment.

The exclusion criteria were as follows: left-handedness according to the Edinburgh Handedness Inventory [70]; severe cognitive impairment $(<10$ points according to the Montreal Cognitive Assessment Scale [20]); sensory aphasia; severe motor aphasia; severe vision impairment preventing execution of visual instructions shown on the computer screen; muscle contracture in the upper extremity (4 points according to the Modified Ashworth Scale [21]).

The withdrawal criteria were as follows: patient refusal to continue participating in the study; development of an acute disease or decompensation of a chronic disease with the risk of a potential impact on the study results (repeated stroke, acute myocardial infarction, non-compensated diabetes, etc.); prescription of systemic muscle relaxants (or changing their dose after inclusion to the study); injection of botulinum toxin agents in muscles of the paretic upper extremity after inclusion of the patient to the study.

The examination data of a patient who signed an informed consent to participate in the study, which met the inclusion criteria, were added to a system for clinical research information support (ImagerySoft, Russia). The participants were assigned identification numbers; then, randomization at a 2:1 ratio was performed to assign a study group (main or control). Patients of the main group were trained with the BCI-exoskeleton technology, while patients of the control group were trained with a technology simulator: their hands were moved by exoskeleton with the same intensity as for the main group but not synchronously with BCI commands. A total of up to 12 training sessions, up to 40 mins, every day (tolerance interval of up to 3 days). Patients in both groups were also provided with kinesiotherapy and massage. Before and after the training course, patients were assessed for movements by upper limb using the Fugl-Meyer (FM) scale and Action Research Arm Test (ARAT). The FM is more versatile and detailed [83] while ARAT is a functional scale which evaluates hand movements required for daily tasks [26].

Until May 2014 totally 245 patients were screened for participation in the study, 71 patients met the inclusion criteria, 11 of them refused to participate in the study after the first or second procedure. Thus, the study includes 60 patients, 46 patients had ischemic stroke, and 14 patients had hemorrhagic stroke. The median 
and $25 \%, 75 \%$ quartiles for stroke duration were $8[3,13]$ months and severity of upper extremity paresis was $4.5[0,30]$ ) scores (ARAT) or $22.5[10,40]$ scores (FM). 42 patients were trained with the BCI (main group), and 18 patients were trained with the simulator (control group). The groups were matched by age, post-stroke duration, and extent of neurological deficit (Mann-Whitney test, p-value $p>0.05$ ). There were also no significant differences in these characteristics among patients from the three clinical centers $(p>0.05)$. Demographic and main baseline data of patients in both groups are shown in Tab. II.

\begin{tabular}{llcc}
\hline Variable & & $\begin{array}{c}\text { Main } \\
(n=42)\end{array}$ & $\begin{array}{c}\text { Control } \\
(n=18)\end{array}$ \\
\hline Age, years & & $58[51,64]$ & $58[52,67]$ \\
\hline Males, $n$ & & $29(69.0 \%)$ & $13(72 \%)$ \\
\hline Months after stroke & & $8[4,13]$ & $6.5[1,13]$ \\
\hline \multirow{2}{*}{ Lesion lateralization } & left & $21(50.0 \%)$ & $13(72.2 \%)$ \\
& right & $21(50.0 \%)$ & $5(27.8 \%)$ \\
\hline \multirow{2}{*}{ Lesion lateralization } & Cortical & $2(4.8 \%)$ & $2(11.1 \%)$ \\
& Subcortical & $18(42.8 \%)$ & $9(50.0 \%)$ \\
& Cortical-subcortical & $22(52.4 \%)$ & $7(38.9 \%)$ \\
\hline ARAT, score & & $4.5[0,31]$ & $7[0,30]$ \\
\hline FM, score & & $27.5[10,40]$ & $12.5[11,49]$ \\
\hline
\end{tabular}

Tab. II Demographic and baseline characteristics of patients in both groups.

The protocol of the rehabilitation procedure was described in Section 2 and in $[14,60,66]$ in more detail. To investigate the rehabilitation effect of the exoskeleton controlled by BCI, we used the hand exoskeleton with two degree of freedom: flexion - extension at the wrist and flexion - extension of hand fingers (Fig. 7). The patients were asked by instruction to imagine extension of hand fingers. Exoskeleton produced extension if BCI recognized the performance of this task and flexion in the opposite case. The amplitudes of flexion and extension were limited not to damage the patient hand. For ten days of training the patients significantly improved the efficiency of BCI control: the median of classification accuracy over the main group increased from $0.41[0.37,0.46]$ to $0.52[0.45,0.65]$ (Mann-Whitney test, $p<0.01)$.

Hand movement imagination was used because, first, the hand is represented in the brain by the large area [16], and second, hand motion is more difficult to restore after stroke and brain trauma than proximal parts of the arm [85]. Thus, the use of BCI based on imaginary of hand movements may be especially beneficial. 


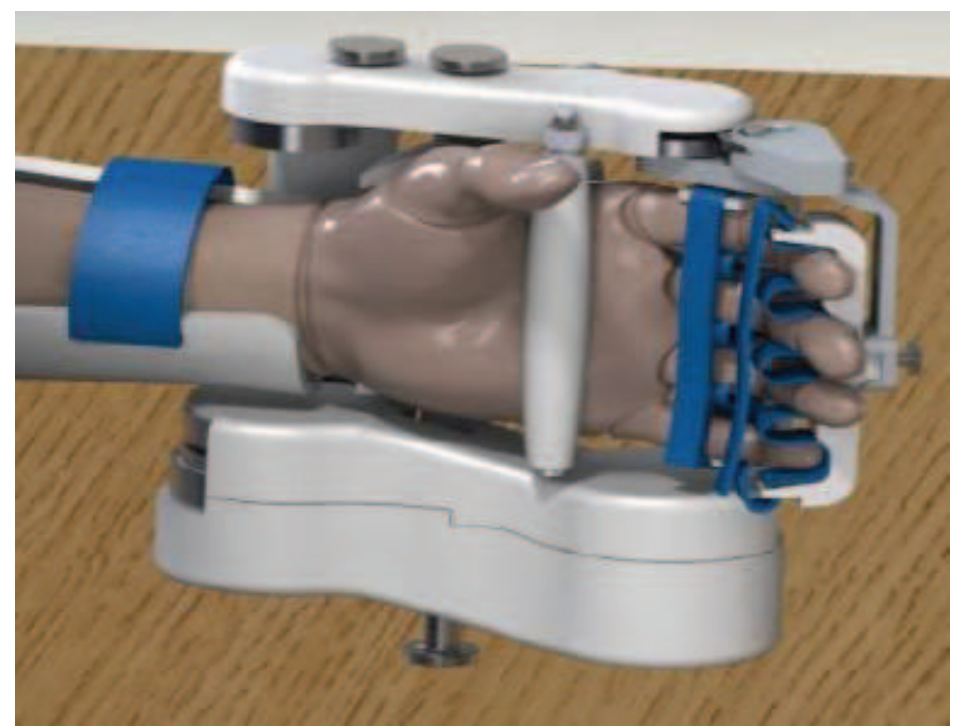

Fig. 7 A human hand exoskeleton with two degrees of freedom (flexion - extension at the wrist and flexion-extension of hand fingers) developed by the company "Android Technique", Russia.

\subsection{Clinical Results}

No significant deterioration in the upper extremity function, according to ARAT or FM, was observed in the patients during the study. Three patients developed a slight headache: 2 patients of the main group (during 2 of 10 trainings in one of them and in the course of 10 trainings in the second patient) and 1 patient of the control group (in 3 of 10 trainings). Most patients of the main group reported fatigue associated with concentration of attention after about 20-30 mins of training. The fatigue was more pronounced in the case of insomnia on the eve of a training, propensity to depression, a large load of other procedures received by the patient before the training, and initial general weakness of a patient. Most patients believed that the sensation of fatigue was related to the training intensity and had a positive attitude towards this effect. Increased blood pressure of 200/100 mm $\mathrm{Hg}$ occurred in one patient of the main group after the third training, which was therapeutically reversed. In general, none of the patients withdrew from the study because of adverse events.

An improvement of the upper extremity motor function was observed in both groups assessed by ARAT and FM (hand-related sections: A-D, H, I). Improvements of the grasp, pinch, and gross movements were assessed by ARAT only in the main group (Tab. III). In this group, a clinically significant improvement of the upper extremity motor function according to ARAT (an increase by 5 points or more) and FM (an increase by 7 points or more, A-D sections) was observed in $30.1 \%$ of patients. The observed positive changes were mainly due to recovery of the hand motor function. In the control group, the percentage of patients with a clinically significant improvement of the hand motor function was only $11.1 \%$. 
Neural Network World 1/2017, 107-137

\begin{tabular}{|c|c|c|c|c|}
\hline Parameter & & $\begin{array}{l}\text { Main } \\
(n=42)\end{array}$ & $\begin{array}{l}\text { Control } \\
(n=18)\end{array}$ & $\begin{array}{l}\text { Possible range } \\
\text { of ARAT scores }\end{array}$ \\
\hline \multirow{3}{*}{ Total scores } & Before & $4.5[0,31]$ & $7[0,30]$ & \multirow{3}{*}{$0-57$} \\
\hline & After & $7[1,43]$ & $9[0,31]$ & \\
\hline & $p$ & $<0.001$ & 0.008 & \\
\hline \multirow{3}{*}{ Grasp } & Before & $1.5[0,14]$ & $0.5[0,12]$ & \multirow{3}{*}{$0-18$} \\
\hline & After & $3.5[0,15]$ & $1.5[0,12]$ & \\
\hline & $p$ & $<0.001$ & 0.345 & \\
\hline \multirow{3}{*}{ Grip } & Before & $5.5[0,8]$ & $1[0,6]$ & \multirow{3}{*}{$0-12$} \\
\hline & After & $1.5\left[\begin{array}{ll}0 & 10\end{array}\right]$ & $2[0,7]$ & \\
\hline & $p$ & $<0.001$ & 0.028 & \\
\hline \multirow{3}{*}{ Pinch } & Before & $0[0,7]$ & $1[0,4]$ & \multirow{3}{*}{$0-18$} \\
\hline & After & $1[0,12]$ & $1[0,5]$ & \\
\hline & $p$ & 0.002 & 0.675 & \\
\hline \multirow{3}{*}{ Gross movement } & Before & $2[0,6]$ & $1.5[0,6]$ & \multirow{3}{*}{$0-9$} \\
\hline & After & $3[1,7]$ & $2[0,6]$ & \\
\hline & $p$ & $<0.001$ & 0.178 & \\
\hline
\end{tabular}

Tab. III Changes in the main clinical indicators in each of the studied groups assessed by ARAT.

Upper extremity function recovery (according to ARAT and FM scores and subscores) did not depend on the stroke duration and patient age either in the BCI-group or in the control group. In each group, a moderate correlation between the extent of arm function recovery (in particular, hand function recovery) and initial severity of a neurological deficit according to ARAT was found $(r=0.4$, $p<0.05)$; but in the main group, a statistically significant improvement of the hand function was observed in subgroups with initially severe, mild and moderate paresis (Tab. IV).

The results of our study are consistent with the data of other controlled studies in this area $[1,2,81]$. It should be noted that, in contrast to our study, patients in the previous studies were pre-screened for the ability to operate MI-based BCI. Another important difference of those studies is a much greater intensity of trainings: 18 hours for the entire course [1,2] compared to our study where the overall training time was 5 hours on average. It was impossible to increase the duration of training because of the limited hospitalization time in Russia. However, three patients were additionally trained due to the next planned hospitalization 6-9 months later. The results of two sequential training courses for them are shown in Fig. 8. According to ARAT scale motor functions worsened to the time of the second rehabilitation for none patients. FM scores (sections C-D) in patient 1 slightly decreased but continued to be higher than before the first course of training. Repeated course of the therapy including BCI training improved motor ability in all three patients, confirming the previous results $[1,2]$ that motor ability indices increase with training duration. 
Frolov A.A. et al.: Principles of motor recovery in post-stroke...

\begin{tabular}{lllc}
\hline Initial paresis severity (FM, sections B-C) & $n$ & & FM scores \\
\hline & & Before & $2[1,6]$ \\
Plegia or severe paresis, $0-12$ points & 28 & After & $2.5[1,8]$ \\
& & $p$ & 0.002 \\
\hline & & Before & $1[1,2]$ \\
Plegia or severe paresis, $0-7$ points & 23 & After & $2[1,6]$ \\
(of 28 previous $0-12$ points) & & $p$ & 0.002 \\
\hline & & Before & $17[14,21]$ \\
Mild or moderate paresis, $13-24$ points & 14 & After & $22[18,24]$ \\
& & $p$ & 0.002 \\
\hline
\end{tabular}

Tab. IV Improvement of the hand motor function (FM scale) in patients of the main group, depending on baseline severity of paresis.

In addition to standard neurological scales FM and ARAT the efficiency of motor recovery was assessed also by recording movement kinematics using electromagnetic spatial tracking systems Flock of Birds, MiniBirds and TrakSTAR (Ascension Technology Corp.) described above. Results obtained for one of the patients of the main group are shown in Fig. 9. Recording was performed by TrakSTAR. Four sensors were mounted at the arm segments, as shown in Fig. 5. The patient was asked to move the arm sequentially in one of seven degrees of mobility periodically with maximal rate. Positions and orientations of joint axes of rotations and angles of rotations around them were calculated, as described in [13].

ARAT

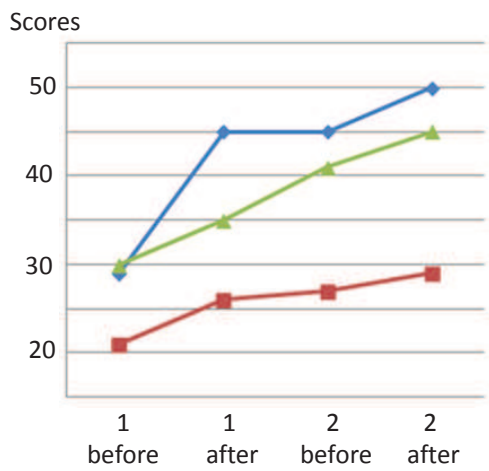

F-M

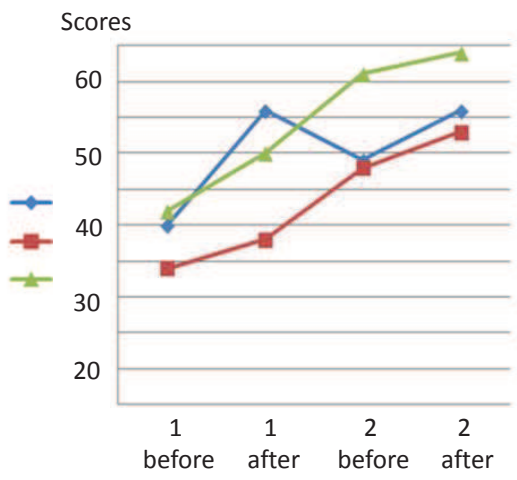

Fig. 8 Indices of motor ability for three patients undergoing the second course of BCI training. 1, 2 (before, after) the indices of the first and second course before and after training. Post-stroke period for the first and second hospitalization: 21 and 30 months for patient 1 (diamond), 9 and 14 months for patient 2 (triangle) and 6 and 12 months for patient 3 (square). 


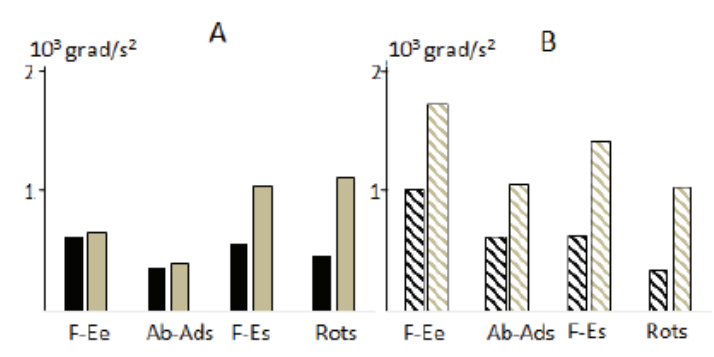

Fig. 9 Maximal values of angular acceleration during periodic arm movement with maximal achievable rate along one of four degrees of mobility: F-Ee - flexion extension in the elbow, Ab-Ads - abduction - adduction in the shoulder, F-Es flexion - extension in the shoulder, Rots - rotation in the shoulder. A-movements by the paretic arm, black bars - before, grey bars - after ten days of training. $B$ movements by the intact arm, black dashed bars - before, grey dashed bars - after ten days of training.

Maximal values of angular accelerations during periodic arm movement with maximal achievable rate along one of four degrees of mobility: F-Ee - flexion extension in the elbow, Ab-Ads - abduction - adduction in the shoulder, F-Es flexion - extension in the shoulder, Rots - rotation in the shoulder. A-movements by the paretic arm, black bars - before, grey bars - after ten days of training. B movements by the intact arm, black dashed bars - before, grey dashed bars - after ten days of training.

Before starting rehabilitation procedure the patient could move the arm only along four degrees of mobility, shown in Fig. 9. Both for paretic and intact arms accelerations essentially increased as a result of training especially in the shoulder joint. Thus, training in distal parts of the arm has essentially improved movements also in proximal parts of the arm. Essential improvement of motor functions in intact arm demonstrates that a stroke usually results in bilateral impairment. FM revealed significant improvement in proximal part of the paretic arm (from 10 to 17), but did not reveal any change in the intact arm (34 before and after training, the maximal FM score for proximal part of the healthy arm is 42). Thus, kinematic recording is much a more precise test to assess movement ability than standard neurological tests.

\section{Conclusions}

Application of BCI is most promising for the creation of a new channel of communication with the outside world of completely paralyzed patients, instead of their natural communication channel, which was lost because it requires muscles activation. Another field of application is motor recovery due to the stimulation of brain plasticity by motor imagery controlled via the biofeedback provided by BCI. This rehabilitation technology seems to be the most efficient if the biofeedback is produced by the movements of the exoskeleton controlled by BCI. In this case, the 
central motor command is reinforced by the peripheral proprioceptive signal concerning its execution. Data on the successful usage of BCI for this task stimulate, besides developing BCI themselves, also the development of anthropomorphic prostheses and exoskeletons, for whose control the biologically appropriate principles seem to be useful, including independent control of eigenmovements, described in Section 3.2. Thus, the development of rehabilitation BCI-exoskeleton technology is a multidisciplinary research which requires the teamwork of experts in neurology, neurophysiology, physics, mathematics, biomechanics and robotics.

The preliminary findings of the blind, controlled study described in the present paper demonstrate that rehabilitation using the BCI-exoskeleton technology increases the percentage of patients with clinically significant recovery in the upper extremity motor function within $2-3$ weeks. An improvement of grasp and pinch of the hand was observed only in the BCI-exoskeleton group. An improvement of the hand motor functions was observed in the subgroups with initially severe, mild to moderate paresis. Therefore, motor deficit severity is not a selection criterion of patients for BCI-exoskeleton training. Furthermore, these trainings are the only method involving the active movement paradigm in patients with plegia and severe paresis. None of the patients withdrew from the study due to a serious adverse event, which means that the technology is safe in general. Since fatigue during training was preceded by insomnia, large exercise load of preceding procedures, propensity to depression, and general weakness, the likelihood of this adverse event can be reduced by selecting the optimal sequence of rehabilitation procedures and surveying the patient about his current state of health and quality of sleep before each training.

In spite of many positive aspects of BCI-exoskeleton technology there were no found statistically significant differences between BCI and control groups in the total evaluation of motor function recovery, which may be due to an insufficient duration of trainings [2], limited in our study by formal administrative reasons. Another possible drawback of our approach is using hand instead of the whole arm exoskeleton. Hand exoskeleton provides the simplest single joint movement. However, it is well known $[8,87]$ that multi-joint goal-directed movements are much more efficient for motor recovery than single joint movements. That is why experiments with the available arm exoskeleton with 7 degrees of freedoms produced by the company "Android Technique", Russia, is the next step of study.

In general, the described principles of motor recovery in the development of the hand and arm exoskeletons and link-up exoskeleton control system to the braincomputer interface are promising field of research which is at the forefront of the development of robotic neurorehabilitation systems.

\section{Acknowledgement}

The work was supported by the Russian Ministry of Education and Science, grant No. RFMEFI60715X0128.

\section{References}

[1] ANG K.K., GUAN C., PHUA K.S., WANG C., ZHOU L., TANG K.Y., JOSEPH G.J., KUAH C.W., CHUA K.S.G. Brain-computer interface-based robotic end ef- 


\section{Neural Network World 1/2017, 107-137}

fector system for wrist and hand rehabilitation: results of a three-armed randomized controlled trial for chronic stroke. Frontiers in Neuroengineering. 2014, 7, 30 p. http://journal.frontiersin.org/article/10.3389/fneng.2014.00030/full, doi: 10.3389/fneng. 2014.00030.

[2] ANG K.K., CHUA K.S., PHUA K.S., WANG C., CHIN Z.Y., KUAH C.W., KUAH C.W., LOW W., GUAN C. A Randomized Controlled Trial of EEG-Based Motor Imagery BrainComputer Interface Robotic Rehabilitation for Stroke. Clinical EEG and Neuroscience. 2015, 46(4), pp. 310-20, doi: 10.1177/1550059414522229.

[3] ALEXANDROV A.V., FROLOV A.A., MASSION J. "Biomechanical analysis of movement strategies in human forward trunk bending. I. Modeling.", Biological Cybernetics. 2001, 84(6), pp. 425-434, doi: 10.1007/PL00007986.

[4] ALEXANDROV A.V., FROLOV A.A., MASSION J. Biomechanichal analysis of movement strategies in human forward trunk bending. II. Experimental study. Biological Cybernetics. 2001, 84(6), pp. 435-443, doi: 10.1007/PL00007987.

[5] AleXandrov A.V., Frolov A.A., HORAK F.B., CARLSON-KUHTA P. PARK S. Feedback equilibrium control during human standing. Biological Cybernetics. 2005, 93(5) pp. 309-322, doi: 10.1007/s00422-005-0004-1.

[6] ALEXANDROV A.V., FROLOV A.A. Closed-loop and open-loop control of posture and movement during human trunk bending. Biological Cybernetics. 2011, 104(6), pp. 425-38, doi: $10.1007 / \mathrm{s} 00422-011-0442-\mathrm{x}$.

[7] AN K.N., JACOBSEN M.C., BERGLUND L.J., CHAO E.Y.S. Application of a magnetic tracking device to kinesiologic studies. Journal of Biomechanics. 1988, 21(7), pp. 613-620, doi: 10.1016/0021-9290(88) 90225-4.

[8] BACH-Y-RITA P. Theoretical and practical considerations in the restoration of function after stroke. Top stroke rehabilitation. 2001, (8), pp. 1-15, doi: 10.1007/s00422-005-0004-1.

[9] BARIN K. Evaluation of a generalized model of human postural dynamics and control in the sagittal plane. Biological Cybernetics, Jan. 1989, 61(1), pp. 37-50, doi: 10.1007/BF00204758.

[10] BASHASHATI H., WARD R.K., BIRCH G.E., BASHASHATI A. Comparing different classifiers in sensory motor brain computer interfaces. PlOS ONE, 2015, 10(6), e0129435, doi: 10.1371/journal.pone. 0129435 .

[11] BERNSTEIN N.A., LATASH M.L., TURVEY M. Dexterity and its development, Taylor 8$\}$ Francis, 1996, pp. 3-244

[12] BIRYUKOVA E.V., ROSCHIN V.Y., FROLOV A.A., IOFFE M.E., MASSION J., DUFOSSE M. Forearm postural control during unloading: anticipatory changes in elbow stiffness Experimental Brain Research. 1999. 124(1), pp. 107-117, doi: 10.1007/s002210050605.

[13] BIRYUKOVA E.V., ROBY-BRAMI A., FROLOV A.A., MOKHTARI M. Kinematics of human arm reconstructed from Spatial Tracking System recordings. Journal of Biomechanics. 2000, 33(8), pp. 985-995, doi: 10.1016/S0021-9290(00)00040-3.

[14] BIRYUKOVVA E.V., PAVLOVA O.G., KURGANSKAYA M.E., BOBROV P.D., TURBINA L.G., FROLOV A.A., MOKIENKO O.A. Recovery of the motor function of the arm with the aid of a hand exoskeleton controlled by a brain-computer interface in a patient with an extensive brain lesion. Human Physiology. 2016, 42(1), pp. 13-23, doi: 10.1134/ S0362119716010035.

[15] BIZZI E., ACCONERO N., CHAPPLE W., HOGAN N. Arm trajectory formation. Experimental Brain Research. 1982, (46), pp. 139-143, doi: 10.1007/BF00238107.

[16] BLUM F., LEISERSON A., HOFSTEDTER L. Mozg, razum i povedenie (A Brain, Mind and Behavior). Mir, Moscow. 1988. 248 p. in Russian.

[17] BOBROV P., FROLOV A.A., CANTOR C., FEDULOVA I., BAKHNYAN M. BrainComputer Interface Based on Generation of Visual Images PLOS ONE. 2011, 6(6). e20674. doi: $10.1371 /$ journal. pone. 0020674 .

[18] BOBROV P.D., KORSHAKOV A.V., ROSHCHIN V.Y., FROLOV A.A. Bayesian classifier for brain-computer interface based on mental representation of movements. I.P. Pavlov Journal of Higher Nervous Activity (cited in PubMed as Zh Vyssh Nerv Deiat I P Pavlova). January 2012, 62(1), pp. 89-99, in Russian. 
Frolov A.A. et al.: Principles of motor recovery in post-stroke...

[19] BOBROV P.D., ISAEV M.R., KORSHAKOV A.V., OGANESYAN V.V., KERECHANIN J.V., POPODKO A.I., FROLOV A.A. Sources of electrophysiological and foci of hemodynamic brain activity most relevant for controlling a hybrid brain-Computer interface based on classification of EEG patterns and near-infrared spectrography signals during motor imagery. Human Physiology. 1 May 2016, 42(3), pp. 241-251, doi: 10.1134/S036211971603004X.

[20] BOCTI C., LEGAUlT V., LEBLANC N., BERGER L., NASREDDinE Z., BEAUliEUBOIRE I., YANEVA K., BOULANGER J.M. Vascular cognitive impairment: most useful subtests of the Montreal Cognitive Assessment in minor stroke and transient ischemic attack. Dementia and geriatric cognitive disorders. 2013, 36(3-4), pp. 154-62. doi: 10.1159/ 000351674.

[21] BOHANNON R.W., SMITH M.B. Interrater reliability of a modified Ashworth scale of muscle spasticity. Physical Therapy. 1987, 67(2), pp. 206-207.

[22] COMPSTON A. From the archives O'BRIEN M. Aids to the investigation of peripheral nerve injuries. Medical Research Council: Nerve Injuries Research Committee. His Majesty's Stationery Office: 1942, 48 p. Fifth edition: Saunders Elsevier: 2010, 70p.) Brain : a journal of neurology. 2010, 133(10), pp. 2838-2844, Book review doi: 10.1093/brain/awq270.

[23] DALY J.J., WOLPAW J.R. Brain-computer interfaces in neurological rehabilitation. The Lancet Neurology. 2008, 7(11), pp. 1032-1043, doi: 10.1016/S1474-4422(08)70223-0.

[24] DELORME A., MAKEIG S. EEGLAB: an open source toolbox for analysis of single-trial EEG dynamics. Journal of Neuroscience Methods. 2004, 134(1), pp. 9-21. doi: 10.1016/j. jneumeth.2003.10.009.

[25] DELORME A., PALME, J., ONTON J., OOSTENVELD R., MAKEIG S. Independent EEG sources are dipolar. PLOS ONE. 2012, 7(2), e30135, doi: 10.1371/journal.pone.0030135.

[26] DOUSSOUlin S.A., RIVAS S.R., CAMPOS S.V. Validation of "Action Research Arm Test" (ARAT) in Chilean patients with a paretic upper limb after a stroke. Revista medica de Chile. 2012, 140(1), pp. 59-65, doi: 10.1186/1743-0003-6-17.

[27] FERRIS D.P. The exoskeletons are here. Journal of NeuroEngineering and Rehabilitation. 2009, 6(17), doi: 10.1186/1743-0003-6-17.

[28] FROLOV A.A., ROSCHIN V.Y., BIRYUKOVA E.V. Adaptive neural network model of multijoint movement control by working point velocity. Neural Network World. 1994, 4(2), pp. 141-156.

[29] FROLOV A.A., DUFOSSE M., RIZEK S., KALADJAN A. On the possibility of linear modeling of the human arm neuromuscular apparatus. Biological Cybernetics. 2000, 82(6), pp. 499-515, doi: 10.1007/s004220050603.

[30] Frolov A.A., PROKOPEnKo R.A., Dufosse M., OUezdou F.B. Adjustment of the human arm viscoelastic properties to the direction of reaching. Biological Cybernetics. 2006, 94(2), pp. 97-109, doi: 10.1007/s00422-005-0018-8.

[31] FROLOV A., HUSEK D., BOBROV P. Comparison of four classification methods for brain computer interface. Neural Network World. 2011, 21(2), pp.101-192, doi: 10.14311/NNW. 2011.21 .007$.

[32] FROLOV A., HUSEK D., BOBROV P., KORSHAKOV A., CHERNIKOVA L., KONOVALOV R., MOKIENKO O. Sources of EEG activity the most relevant to performance of brain-computer interface based on motor imagery. Neural Network World. 2012, 22(1), pp. 21-37, doi: 10.14311/NNW.2012.22.002.

[33] FROLOV A.A., BIRYUKOVA E.V., BOBROV P.D., MOKIENKO O.A., PLATONOV A.K., PRYANICHNIKOV V.E., CHERNIKOVA L.A. Principles of neurorehabilitation based on the brain-computer interface and biologically adequate control of the exoskeleton. Human Physiology. 2013, 39(2), pp. 196-208, doi: 10.1134/S0362119713020035.

[34] FROLOV, A.A., HUSEK D., BOBROV P., MOKIENKO O., TINTERA J. Sources of electrical brain activity most relevant to performance of brain-computer interface based on motor imagery. Brain-Computer Interface Systems-Recent Progress and Future Prospects. 2013, pp.175-193, doi: 10.5772/55166. 


\section{Neural Network World 1/2017, 107-137}

[35] FROLOV A.A., HUSEK D., SILCHENKO A.V., TINTERA J., RYDLO J. The changes in the hemodynamic activity of the brain during motor imagery training with the use of brain-computer interface. Human Physiology. 2016, 42(1), pp. 1-12, doi: 10.1134/ S0362119716010084.

[36] FROLOV A.A., BOBROV P.D. Reaching model perurbed. RMP.wmv [video]. 2016-10-24 [accessed 2016-10-27]. Available from: http://www.cs.cas.cz/dusan/BCI/

[37] FROLOV A.A., BOBROV D.A., BOBROV P.D. Reaching model unperturbed. RMU.wmv [video]. 2016-10-24 [accessed 2016-10-27]. Available from: http://www.cs.cas.cz/dusan/BCI/

[38] FROLOV A.A., BOBROV D.A., BOBROV P.D. Reaching exoskeleton. REX.mp4 [video]. 2016-10-24 [accessed 2016-10-27]. Available from: http://www.cs.cas.cz/dusan/BCI/

[39] GAWTHROP P., LORAM I., LAKIE M. Predictive feedback in human simulated pendulum balancing. Biological Cybernetics. 2009, 101(2), pp. 131-146, doi: 10.1007/ s00422-009-0325-6.

[40] GEORGOPOUlos A.P., KAlASKA J.F., CRUTCHER M.D., CAMINITI R., MASSEY J.T. On the relations between the direction of two-dimensional (2D) arm movements and cell discharge in primate motor cortex. Journal of Neuroscience. 1982, (2), pp.1527-1537.

[41] GOMI H., KAWATO M. Equilibrium-point control hypothesis examined by measured arm stiffness during multijoint movement. Science. 1996, (272), pp. 117-120, doi: 10.1126/ science.272.5258.117.

[42] GOMI H., KAWATO M. Human arm stiffness and equilibrium-point trajectory during multi-joint movement. Biological Cybernetics. 1998, (76), pp. 163-171, doi: 10.1007/ s004220050329.

[43] GRECH R., CASSAR T., MUSCAT J., CAMILLERI K.P., FABRI S.G., ZERVAKIS M., XANTHOPOULOS P., SAKKALIS V., VANRUMSTE B. Review on solving the inverse problem in EEG source analysis. Journal of Neuroengineering and Rehabilitation. 2008, 5(1), 33p., doi: 10.1186/1743-0003-5-25.

[44] GREZES J., DECETY J. Functional anatomy of execution, mental simulation, observation, and verb generation of actions: a meta-analysis. Human Brain Mapping. 2001, 12(1), pp. 119, doi: 10.1002/1097-0193(200101) 12:1<1: : AID-HBM10>3.0.CO;2-V.

[45] GRINYAGIN I.V., BIRYUKOVA E.V., MAIER M.A. Kinematic and dynamic synergies of human precision grip movement. Journal of Neurophysiology. 2005, 94(4), pp.2284-2294, doi: $10.1152 / j n .01310 .2004$

[46] GUGER C., EDLINGER G., HARKAM W., NIEDERMAYER I., PFURTSCHELLER G. How many people are able to operate an EEG-based brain-computer interface (BCI). IEEE Transactions on Neural Systems and Rehabilitation Engineering. 2003, 11(2), pp.145-147, doi: 10.1109/TNSRE. 2003.814481.

[47] HetU S., GREGOIRE M., SAIMPONT A., COLL M.P., EUGENE F., MiCHON P.E., JACKSON P.L. The neural network of motor imagery: an ALE meta-analysis. Neuroscience E6 Biobehavioral Reviews. 2013, 37(5), pp.930-949, . doi: 10.1016/j .neubiorev . 2013.03.017.

[48] HINGTEN B., McGUIRE J.R., WANG M., HARRIS G.F. An upper extremity kinematic model for evaluation of hemiparetic stroke. Journal of Biomechanics. 2006, 39(4), pp. 681688, doi: $10.1016 / j \cdot j$ biomech.2005.01.008.

[49] HYVARINEN A., KARHUNEN J., OJE E. Independent component analysis. New-York: John Wiley \& Sons. 2001. 476 p. Errata avilable from: http://research.ics.aalto.fi/ica/book/errata.html (last viewed 12.10.2016), doi: 10.1002/ 0471221317.

[50] JARRASSE N., PROIETTI T., CROCHER V., ROBERTSON J., SAHBANI A., MOREL G., ROBY-BRAMI A. Robotic exoskeletons: a perspective for the rehabilitation of arm coordination in stroke patients. Front. Hum. Neurosci. 2014, 8 article 947 13p. doi: 10. 3389/fnhum. 2014.00947.

[51] JEANNEROD M. The representing brain: Neural correlates of motor intention and imagery. Behavioral and Brain Sciences. 1994, 17(02), pp. 187-202, doi: 10.1017/S0140525X00034026. 
Frolov A.A. et al.: Principles of motor recovery in post-stroke...

[52] JEANNEROD M. Neural simulation of action: a unifying mechanism for motor cognition. Neuroimage. 2001, 14(1), pp. S103-S109, doi: 10.1006/nimg.2001.0832.

[53] JONES S.R., KERR C.E., WAN Q., PRITCHETT D.L., HAMALAINEN M. MOORE C.I Cued spatial attention drives functionally relevant modulation of the mu rhythm in primary somatosensory cortex. J. Neurosci. 2010, 30(41), pp. 13760-13765, doi: 10.1523/JNEUROSCI. $2969-10.2010$.

[54] KACHENOURA A., ALBERA L., SENHADJI L., COMON P. ICA: a potential tool for BCI systems. IEEE Signal Processing Magazine. 2008, 25(1), pp. 57-68, doi: 10.1523/ JNEUROSCI . 2969-10.2010.

[55] KAUKORANTA E., HAMALAINEN M., SARVAS J., HARI R. Mixed and sensory nerve stimulations activate different cytoarchitectonic areas in the human primary somatosensory cortex SI. Exp. Brain Res. 1986, 63(60), pp. 60-66, doi: 10.1007/BF00235646.

[56] KAWATO M. Internal models for motor control and trajectory planning. Current Opinion in Neurobiology. 1999, 9 (6), pp. 718-727, doi: 10.1016/S0959-4388(99) 00028-8.

[57] KOHAVI R., PROVOST F. Glossary of terms. Machine Learning. (Special Issue of Applications of Machine Learning and the Knowledge Discovery Proceses.) 1998, 30(2/3), pp. 271274 .

[58] KONRAD P., SHANKS T. Implantable brain computer interface: challenges to neurotechnology translation. Neurobiology Disorders. 2010, 38(3), pp. 369-375, doi: 10.1016/j.nbd. 2009.12.007.

[59] KORENEV G.V. Tselenapravlennaya mekhanika upravlyaemykh manipulyatorov. (PurposeOriented Mechanics of Controlled Manipulators), Moscow: Nauka, 1979, in Russian.

[60] KOTOV S.V., TURBINA L.G., BOBROV P.D., FROLOV A.A., PAVLOVA O.G., KURGANSKAYA M.E., BIRYUKOAVA E.V. Rehabilitation of Stroke Patients with a Bioengineered "Brain-Computer Interface with Exoskeleton" System. Neuroscience and Behavioral Physiology. 2016, 46(5), pp.518-522, doi: 10.1007/s11055-016-0270-5.

[61] KWAKKEL G., WAGENAAR R.C., TWISK J.W., LANKHORST G.J., KOETSIER J.C. Intensity of leg and arm training after primary middle-cerebral-artery stroke: a randomised trial. The Lancet Neurology. 1999, 354(9174), pp. 191-196. doi: 10.1016/S0140-6736(98) 09477-X.

[62] LANGHORNE P., COUPAR F., POLLOCK A. Motor recovery after stroke: a systematic review. The Lancet Neurology. 2009, 8(8), pp. 741-54, doi: 10.1016/S1474-4422 (09) 70150-4.

[63] LEBEDEV M.A., NICOLELIS M.A.L. Brain-machine interfaces: past, present and future. Trends in Neurosciences. 2006, 29(9), pp. 536-546, doi: 10.1016/j.tins.2006.07.004.

[64] MARCHAL-CRESPO L., REINKENSMEYER D.J. Review of control strategies for robotic movement training after neurologic injury. Journal of NeuroEngineering and Rehabilitation. 2009, 6(20), Available from: http://www.jneuroengrehab.com/content/6/1/20 doi: 10.1186/ 1743-0003-6-20.

[65] MILlAN J. del R., MOURINO J., MARCIANI M.G., BABILONI F., TOPANI F., CANALE I., HEIKKONEN J., KASKI K. Adaptive brain interfaces for physically-disabled people. 2nd Annual Int.Conf. of the IEEE Engineering in Medicine and Biology Science. Hong Kong, 1998.

[66] MOKIENKO O.A., LYUKMANOV R.K., CHERNIKOVA L.A., SUPPONEVA N.A., PIRADOV M.A., FROLOV A.A. Brain-computer interface: The first experience of clinical use in Russia. Human Physiology. 2016, 42(1), pp. 24-31, doi: 10.1134/S0362119716010126.

[67] NEUPER C., PFURTSCHELLER G. Motor imagery and ERD. Event-related desynchronization. Handbook of Electroencephalography and Clinical Neurophysiology. 1999, 6, pp. 303-325.

[68] NEUPER C., SCHERER R., REINER M., PFURTSCHELLER G. Imagery of motor actions: differential effects of kinesthetic and visual-motor mode of imagery in single-trial EEG. Cognitive Brain Research. 2005, 25(3), pp. 668-677, doi: 10.1016/j.cogbrainres.2005.08.014.

[69] NUDO R.J., MILLIKEN G.W., JENKINS W.M., MERZENICH M.M. Use-dependent alterations of movement representations in primary motor cortex of adult squirrel monkeys. Journal of Neuroscience. 1996, 16(2), pp. 785-807. 


\section{Neural Network World 1/2017, 107-137}

[70] OLDFIELD R.C. The assessment and analysis of handedness: the Edinburgh inventory. Neuropsychologia. 1971, 9(1), pp. 97-113. 10.1016/0028-3932(71)90067-4.

[71] ONTON, J., WESTERFIELD, M., TOWNSEND, J., \& MAKEIG, S. Imaging human EEG dynamics using independent component analysis. Neuroscience \& Biobehavioral Reviews. 30(6), 2006 pp.808-822, doi: 10.1016/j.neubiorev. 2006.06.007.

[72] PAOLUCCI S., ANTONUCCI G., GRASSO M.G., MORELlI D., TROISI E., COIRO P., BRAGONI M. Early versus delayed inpatient stroke rehabilitation: a matched comparison conducted in Italy. Archives of Physical Medicine and Rehabilitation. 2000, 81(6), pp. 695700, doi: 10.1016/S0003-9993(00) 90095-9.

[73] PARK S., HORAK F.B., KUO A.D. Postural feedback responses scale with biomechanical constraints in human standing. Exp. Brain Res. 2004, 154(4), pp. 417-427, doi: 10.1007/ s00221-003-1674-3.

[74] PETERKA R.J. Sensorimotor integration in human postural control. Journal of Neurophysiology. 2002, 88(3), pp. 1097-1118.

[75] PETERKA R.J. Comparison of human and humanoid robot control of upright stance. Journal of Physiology. Paris 2009, 103(3-5), pp. 149-158, doi: 10.1016/j.jphysparis.2009.08. 001.

[76] PFURTSCHELleR G., BERGHOLD A. Patterns of cortical activation during planning of voluntary movement. Electroencephalography and Clinical Neurophysiology. 1989, 72(3), pp.250-258. doi: 10.1016/0013-4694(89) 90250-2.

[77] PFURTSCHELLER G. EEG event-related desynchronization (ERD) and event related synchronization (ERS) In: eds.NIEDERMEYER E., LOPES da SILVA F.H. Electroencephalography: basic principles, clinical applications and related fields. 4th ed. Baltimore, MD: Williams and Wilkins. 1999, pp. 958-967.

[78] PFURTSCHELlER G., LOPES da SILVA F.H. Event-related EEG/MEG synchronization and desynchronization: basic principles. Clinical Neurophysiology. 1999, 110. pp. 1842-1857. doi: $10.1016 / \mathrm{S} 1388-2457$ (99) 00141-8.

[79] PFURTSCHELLER G., NEUPER C. Motor imagery and direct brain-computer communication. Proceedings of the IEEE. 2001, 82(7), pp. 1123-1134, doi: 10.1109/5.939829.

[80] PROKOPENKO R.A., FROLOV A.A., BIRYUKOVA E.V., ROBY-BRAMI A. Assessment of the accuracy of a human arm model with seven degrees of freedom. Journal of Biomechanics. 2001, 34(2), pp. 177-185, doi: 10.1002/ana.23879.

[81] RAMOS-MURGUiALDAY A., BROETZ D., REA M., LAER L., YILMAZ O., BRASIL F.L., LIBERATI G, CURADO M.R., GARCIA-COSSIO E., VYZIOTIS Y., CHO W., AGOSTINI M., SOARES E., SOEKADAR S., CARIA A., COHEN L.G., BIRBAUMER N. Brain-machine interface in chronic stroke rehabilitation: a controlled study. Annals of Neurology. 2013, 74(1), pp. 100-108, doi: 10.1002/ana.23879.

[82] ROUGEUL-BUSER A., BUSER P. Rhythms in the alpha band in cats and their behavioural correlates. International Journal of Psychophysiology. 1997, 26, pp. 191-203, doi: 10.1016/ S0167-8760 (97) 00764-2.

[83] SANFORD J., MORELAND J., SWANSON L.R., STRATFORD P.W., GOWLAND C. Reliability of the Fugl-Meyer assessment for testing motor performance in patients following stroke. Physical Therapy. 1993, 73(7), pp. 447-454.

[84] SOLODKIN A., HLUSTIK P., CHEN E.E., SMALL S.L. Fine modulation in network activation during motor execution and motor imagery. Cerebral Cortex. 2004, 14(11), pp. 1246-1255, doi: oi.org/10.1093/cercor/bhh086.

[85] SHELTON F.N.A.P., REDING M.J. Effect of lesion location on upper limb motor recovery after stroke. Stroke. 2001, 32(1), pp. 107-112, doi: doi.org/10.1161/01.STR.32.1.107.

[86] SONTAG E.D. Mathematical control theory: Deterministic finite dimensional systems. Springer Science \& Business Media. 1998.

[87] TAUB E., USWATTE G., ELBERT T. New treatments in neurorehabilitation founded on basic research. Nature Reviews. Neuroscience. 2002, 3(3), pp. 228-236, doi.org/10.1038/nrn754. 
Frolov A.A. et al.: Principles of motor recovery in post-stroke...

[88] THRIFT A.G., CARDILHAC D.A., THAYABARANATHAN T., HOWARD G., HOWARD V.J., ROTHWELL P.M., DONNAN G.A. Global stroke statistics. International Journal of Stroke. 2014, 9(1), pp. 6-18.

[89] VIDAL J.J. Towards direct brain-computer communication. Annual Review of Biophysics and Bioengineering. 1973, 2, pp. 157-180, doi: 10.1146/annurev.bb.02.060173.001105.

[90] VIDAL J.J. Real-time detection of brain events in EEG. IEEE Proceedings. Special issue on Biological Signal Processing and Analysis. 1977, (65), pp. 633-664, doi: 10.1109/proc. 1977. 10542.

[91] WEISS P. Self differentiation of basic patterns of coordination. Comparative Psychological Monograph. Baltimore, Md: The Williams \& Wilkins Company 1941, 17(4) serial No.88, pp. 1-96.

[92] WINTER D.A. Biomechanics and motor control of movement. New York: Wiley Interscience, 2nd ed. 1990.

[93] WOLPAW J.R. Brain-computer interfaces as new brain output pathways. Journal of Physiology. 2007, (579), pp. 613-619, doi: 10.1113/jphysiol.2006.125948.

[94] WOLPAW J.R, MCFARLAND D.J. Control of two-dimensional movement signal by a noninvasive brain-computer interface in humans. Proceedings of the National Academy of Sciences. USA, 2004, 101(51). pp. 17849-17854, doi: 10.1073/pnas.0403504101. 